\title{
Multi-granulation Pythagorean fuzzy decision-theoretic rough sets based on inclusion measure and their application in incomplete multi-source information systems
}

\author{
Prasenjit Mandal $^{1}$ (D) A. S. Ranadive ${ }^{2}$
}

Received: 29 August 2018 / Accepted: 27 December 2018 / Published online: 9 January 2019

(c) The Author(s) 2019

\begin{abstract}
Multi-granulation rough sets (MGRSs) and decision-theoretic rough sets (DTRSs) are two important and popular generalizations of classical rough sets. The combination of two generalized rough sets have been investigated by numerous researchers in different extensions of fuzzy settings such as interval-valued fuzzy sets (IVFSs), intuitionistic fuzzy sets (IFSs), bipolar-valued fuzzy sets (BVFSs), etc. Pythagorean fuzzy (PF) set is another extension of fuzzy set, which is more capable in comparison to IFS handle vagueness in real world. However, few studies have focused on the combination of the two rough sets in PF settings. In this study, we combine the two generalized rough sets in PF settings. First, we introduce a type of PF subset (of subset of the given universe) of the PF Set (of the given universe). Then we establish two basic models of multi-granulation PF DTRS (MG-PF-DTRS) of PF subset of the PF set based on PF inclusion measure within the framework of multi-granulation $\mathrm{PF}$ approximation space. One model is based on a combination of PF relations (PFRs) and the construction of approximations with respect to the combined PFR. By combining PFRs through intersection and union, respectively, we construct two models. The other model is based on the construction of approximations from PFRs and a combination of the approximations. By using intersection and union to combine the approximations, respectively, we again get two models. As a result, we have total four models. Further for different constraints on parameters, we obtain three kinds of each model of the MG-PF-DTRSs. Then, their principal structure, basic properties and uncertainty measure methods are investigated as well. Second, we give a way to compute PF similarity degrees between two objects and also give a way to compute PF decision-making objects from incomplete multi-source information systems (IMSISs). Then we design an algorithm for decision-making to IMSISs using MG-PFDTRSs and their uncertainty measure methods. Finally, an example about the mutual funds investment is included to show the feasibility and potential of the theoretic results obtained.
\end{abstract}

Keywords Pythagorean fuzzy set · Pythagorean fuzzy inclusion measure · Multi-granulation Pythagorean fuzzy decision-theoretic rough set $\cdot$ Incomplete multi-source information system

\section{Introduction}

In light of Bayesian decision procedure [4], decision theoretic rough set (DTRS) model was proposed by Yao and Wong [35] to analyze the noisy data by considering the tolerance of

Prasenjit Mandal

prasenjitmandal08@yahoo.com

A. S. Ranadive

asranadive04@yahoo.co.in

1 Bhalukdungri Jr. High School, Raigara, Purulia, WB 723153, India

2 Department of Pure and Applied Mathematics, Guru Ghasidas University, Bilaspur, CG, India classification error. Since then the DTRS model has found its applications in various theoretical and practical fields, and it has produced many god results [2,15-17,20,25].

However, the DTRS model cannot deal with numerical data directly. To overcome this disadvantage, researchers used tolerance relations [21], similarity measures [23], domains relations [3], covering [33], inclusion measures [38], fuzzy relations $[32,41,42]$, fuzzy preference relations [26], interval-valued fuzzy preference relations [29], intuitionistic fuzzy relations $[19,40]$, intuitionistic fuzzy inclusion measure [13,13], bipolar-valued fuzzy relations [24,28] in place of equivalence relations.

To handle all types of real data, Yager proposed the concepts of Pythagorean fuzzy set (PFS) [34], which are 
more powerful than intuitionistic fuzzy sets (IFSs) [1] for dealing with the uncertain information in decision-making procedures. For example, if a decision maker gives the membership degree and non-membership degree as 0.8 and 0.6 , respectively, then it is only valid for PFS. Fortunately, PFSs generalize the concept of IFSs and the corresponding operational laws, which have been successfully applied to some complex practical decision-making situations, e.g., road-building projects [11], selection of the optimal production strategy [12] and group decision-making problems [8]. Besides, Zhang and Ren [37] investigated Pythagorean fuzzy multigranulation rough set over two universes and its applications in merger and acquisition. Liang et al. [18] gave a method of three-way decisions using ideal TOPSIS solutions on Pythagorean fuzzy informations. Mandal and Ranadive [25] studied decision-theoretic rough sets under Pythagorean fuzzy information.

From the aforementioned literature it is clear that PFSs provide us a novel evaluation format to measure the fuzzy environment, especially when we utilize the positive and negative sides to depict a question $[1,19]$. Based on the Pythagorean fuzzy environment, we introduce PFSs into multi-granulation rough set (MGRS) and decision-theoretic rough set (DTRS), which are two important and popular extended type of Pawlak's classical rough sets [30] and established multi-granulation Pythagorean fuzzy decisiontheoretic rough sets (MG-PF-DTRS) based on Pythagorean fuzzy inclusion measure. First, we introduce a type of Pythagorean fuzzy subset (of subset of the given universe) of the Pythagorean fuzzy Set (of the given universe). Then we establish two basic models of multi-granulation Pythagorean fuzzy decision-theoretic rough set (MG-PFDTRS) of Pythagorean fuzzy subset of the PFS based on Pythagorean fuzzy inclusion measure within the framework of multi-granulation Pythagorean fuzzy approximation space. One model is based on a combination of PF relations (PFRs) and the construction of approximations with respect to the combined PFR. By combining PFRs through intersection and union, respectively, we construct two models. The other model is based on the construction of approximations from PFRs and a combination of the approximations. By using intersection and union to combine the approximations, respectively, we again get two models. As a result, we have total four models. For different constraints on parameters, we obtain three kinds of each model of the MG-PF-DTRSs. Then, their principal structure, basic properties and uncertainty measure methods are investigated as well. Second, we suggest a decision-making process for incomplete multi-source information systems (IMSISs) using these theoretic results about MG-PF-DTRSs. In this process, we encounter two challenges: (1) How to find the similarity degrees between two objects from IMSISs in the Pythagorean fuzzy settings and (2) how to obtain the
Pythagorean fuzzy decision-making objects from IMSISs. To meet out these issues, we first adopt the method of Liu et al. [23] to obtain the unknown value of the given objects with respect to the given attribute. Then we give the method to find the similarity degree between two objects in the Pythagorean fuzzy setting and construct an algorithm for obtaining Pythagorean fuzzy decision-making objects. Having solved these two issues, we design an algorithm for decision-making to IMSISs using MG-PFDTRSs and their uncertainty measure methods. In this algorithm, we address the problem if $X_{1}, X_{2}, \ldots, X_{r} \subseteq U$ ( $U$ is the finite universe of discourse) such that $\left|X_{1}\right|=\left|X_{2}\right|=\cdots=\left|X_{r}\right|$, then find the best $X_{i}(1 \leq i \leq r)$, where the elements of $X_{i}$ are selected randomly. To solve this problem, first we have the IMSIS for the given alternatives with respect to the considered attributes. Second, we compute PFS from IMSIS and PFRs from each sub-information source from IMSIS of $U$. Then, we derive the PFS and PFRs for each $X_{i}$. Third, we obtain the approximation results for each type MG-PFDTRSs for each $X_{i}$. We see that the obtained results are not entirely consistent. For this reason we also suggest several methods of uncertainty measure such as accuracy, approximation degree and approximation quality for four types of MG-PF-DTRSs. Fourth, we obtain best $X_{i}$ according to the higher accuracy, approximation degree and approximation quality. This is our main objective. In comparison to existing results $[13,14]$, our model has several advantages as listed below:

1. our model can deal with both intuitionistic fuzzy and Pythagorean fuzzy information instead of only intuitionistic fuzzy information;

2. our model can deal with complete and incomplete multisource information systems instead of only complete multi-source information systems;

3. instead of assuming a fuzzy decision-making object as many researchers do, we give a method to find it.

As far as organization of this paper is concerned we give some necessary concepts of PFSs in "Preliminaries". In "MG-PF-DTRSs based on inclusion measure", we propose a common framework of inclusion measure based on MG-PF-DTRSs and study four types of MG-PF-DTRSs which are constructed using the Pythagorean fuzzy inclusion measure. The uncertainties of the proposed four types of MG-PF-DTRSs are measured in "Uncertainty measures". In "Decision-making to incomplete multi-source information systems using MG-PF-DTRSs", we apply our theoretical results to decision-making in IMSIS. An example about selection of mutual funds is also included in this section to show the feasibility and potential of our proposed decision-making approach. "Conclusions" is the concluding section. 


\section{Preliminaries}

In this section, we present some basic concepts and terminology used throughout the paper.

Definition 1 (Peng et al. [39]) Let $U$ be a universe of discourse. A PFS $A$ in $U$ is given by

$$
\begin{aligned}
A & =\left\{\left\langle x, \mu_{A(U)}(x), v_{A(U)}(x)\right\rangle \mid x \in U\right\} \\
& =\sum_{x \in U} \frac{\left\langle\mu_{A}(x), v_{A}(x)\right\rangle}{x}
\end{aligned}
$$

where $\mu_{A}: U \rightarrow[0,1]$ denotes the degree of membership and $v_{A}: U \rightarrow[0,1]$ denotes the degree of nonmembership of the element $x \in U$ for the set $A$, respectively, with the condition that $0 \leq \mu_{A}^{2}(x)+v_{A}^{2}(x) \leq 1$. The degree of indeterminacy $\pi_{A}(x)$ is given by $\sqrt{1-\mu_{A}^{2}(x)-v_{A}^{2}(x)}$. For convenience, Zhang and Xu [39] called $\left\langle\mu_{A}(x), \nu_{A}(x)\right\rangle \mathrm{a}$ Pythagorean fuzzy number (PFN) denoted by $A=\left\langle\mu_{A}, v_{A}\right\rangle$.

If $X \subseteq U$, we define a Pythagorean fuzzy subset of $X$ of the PFS of $U$ in the following way:

$A(X)= \begin{cases}\left\langle\mu_{A}(x), v_{A}(x)\right\rangle & \text { if } x \in X, \\ \langle 0,1\rangle & \text { if } x \notin X,\end{cases}$

which may also denoted by

$$
\begin{aligned}
A(X) & =\left\{\left\langle x, \mu_{A(X)}(x), v_{A(X)}(x)\right\rangle \mid x \in X \subseteq U\right\} \\
& =\sum_{x \in X \subseteq U} \frac{\left\langle\mu_{A(X)}(x), v_{A(X)}(x)\right\rangle}{x} .
\end{aligned}
$$

In this case the PFN is denoted by $A(X)=\left\langle\mu_{A(X)}, v_{A(X)}\right\rangle$. The complement of $A(X)$ is denoted by $A\left(X^{c}\right)$ and defined as

$$
A\left(X^{c}\right)= \begin{cases}\left\langle\mu_{A(X}(x), v_{A}(x)\right\rangle & \text { if } x \notin X . \\ \langle 0,1\rangle & \text { if } x \in X .\end{cases}
$$

For example, let $A=\left\{\left\langle x_{1}, 0.9,0.3\right\rangle,\left\langle x_{2}, 0.4,0.7\right\rangle\right.$, $\left\langle x_{3}, 0.8,0.4\right\rangle,\left\langle x_{4}, 0.7,0.2\right\rangle,\left\langle x_{5}, 0.7,0.6\right\rangle,\left\langle x_{6}, 0.9,0.2\right\rangle$, $\left.\left\langle x_{7}, 0.7,0.5\right\rangle,\left\langle x_{8}, 0.8,0.2\right\rangle,\left\langle x_{9}, 0.5,0.7\right\rangle,\left\langle x_{10}, 0.7,0.6\right\rangle\right\}$ be a PFS. If $X=\left\{x_{4}, x_{7}, x_{8}, x_{10}\right\} \subseteq U$, then $A(X)=$ $\left\{\left\langle x_{1}, 0,1\right\rangle,\left\langle x_{2}, 0,1\right\rangle,\left\langle x_{3}, 0,1\right\rangle,\left\langle x_{4}, 0.7,0.2\right\rangle,\left\langle x_{5}, 0,1\right\rangle\right.$, $\left\langle x_{6}, 0,1\right\rangle,\left\langle x_{7}, 0.7,0.5\right\rangle,\left\langle x_{8}, 0.8,0.2\right\rangle,\left\langle x_{9}, 0,1\right\rangle,\left\langle x_{10}, 0.7\right.$, $0.6\rangle\}$ and $A\left(X^{c}\right)=\left\{\left\langle x_{1}, 0.9,0.3\right\rangle,\left\langle x_{2}, 0.4,0.7\right\rangle,\left\langle x_{3}, 0.8\right.\right.$, $0.4\rangle,\left\langle x_{4}, 0,1\right\rangle,\left\langle x_{5}, 0.7,0.6\right\rangle,\left\langle x_{6}, 0.9,0.2\right\rangle,\left\langle x_{7}, 0,1\right\rangle$, $\left.\left\langle x_{8}, 0,1\right\rangle,\left\langle x_{9}, 0.5,0.7\right\rangle,\left\langle x_{10}, 0,1\right\rangle\right\}$.

Throughout this paper by $P F S(U)$ we mean the set of all PFSs defined on $U$.

Definition 2 (Peng et al. [31]) If $A, B \in P F S(U)$, then
1. $A^{c}=\left\{\left\langle x, v_{A}(x), \mu_{A}(x)\right\rangle \mid x \in U\right\}$;

2. $A \subseteq B$ if $\forall x \in U, \mu_{A}(x) \leq \mu_{B}(x)$ and $v_{A}(x) \geq v_{B}(x)$;

3. $A=B$ iff $\forall x \in U, \mu_{A}(x)=\mu_{B}(x)$ and $v_{A}(x)=v_{B}(x)$;

4. $\Phi_{A}=\{\langle x, 1,0\rangle \mid x \in U\}$;

5. $\emptyset_{A}=\{\langle x, 0,1\rangle \mid x \in U\}$

6. $A \cap B=\left\{\left\langle x, \mu_{A}(x) \wedge \mu_{B}(x), v_{A}(x) \vee v_{B}(x)\right\rangle \mid x \in\right.$ $U$;

7. $A \cup B=\left\{\left\langle x, \mu_{A}(x) \vee \mu_{B}(x), v_{A}(x) \wedge v_{B}(x)\right\rangle \mid x \in\right.$ $U\}$.

Thus it is clear that if $X \subseteq U$, then $A(X), A\left(X^{c}\right) \subseteq A$.

Definition 3 (Zhang et al. [36]) A Pythagorean fuzzy relation $R$ on $U$ is a PFS on $U \times U$. That is, $R$ is expressed by

$R=\left\{\left\langle(x, y), \mu_{R}(x, y), v_{R}(x, y)\right\rangle \mid(x, y) \in U \times U\right\}$,

where $\mu_{R}: U \times U \rightarrow[0,1]$ and $v_{R}: U \times U \rightarrow[0,1]$ satisfy $0 \leq \mu_{R}^{2}(x, y)+v_{R}^{2}(x, y) \leq 1$ for all $(x, y) \in U \times U$.

A Pythagorean fuzzy relation $R$ on $U$ is denoted by $R(U)$ in this paper. If $X \subseteq U$, we define a Pythagorean fuzzy relation $R(U \times X)$ on $U \times X$ as follows:

$R(U \times X)= \begin{cases}\left\langle\mu_{R}(x, y), v_{A}(x, y)\right\rangle & \text { if } x \in U, y \in X, \\ \langle 0,1\rangle & \text { if } x \in U, y \notin X .\end{cases}$

For example, let $U=\left\{x_{1}, x_{2}, x_{3}, x_{4}, x_{5}\right\}$ and the Pythagorean fuzzy relation on $U$ be as given in Table 1 and $X=\left\{x_{3}, x_{5}\right\} \subseteq U$; then the Pythagorean fuzzy relation on $U \times X$ will be as given in Table 2 .

The Pythagorean fuzzy inclusion measure is also called the Pythagorean fuzzy subsethood measure, which indicates the degree to which one PFS is contained in another PFS. Peng et al. [31] provided a simple definition of the Pythagorean fuzzy inclusion measure as follows:

Definition 4 (Peng et al. [31]) Let $A, B$ and $C$ be three PFSs on $U$. An inclusion measure $I(A, B)$ is a mapping $I: \operatorname{PFS}(U) \times \operatorname{PFS}(U) \rightarrow[0,1]$, possessing the following properties:

1. $0 \leq I(A(U), B(U)) \leq 1$;

2. $I(A, B)=1$ iff $A \subseteq B$;

3. $I(A, B)=0$ iff $A=\Phi_{A}, B=\emptyset_{B}$;

4. If $A \subseteq B \subseteq C$, then $I(C, A) \leq I(B, A)$ and $I(C, A) \leq$ $I(C, B)$.

We provide an inclusion measure on $\operatorname{PFS}(U)$ on the basis of the Theorems 3.7(10) and 3.22 presented by Peng et al. [31].

Definition 5 (Peng et al. [31]) For $A, B \in \operatorname{PFS}(U)$, an inclusion measure $I$ on $\operatorname{PFS}(U)$ can be defined as follows: 
Table 1 The Pythagorean fuzzy relation on $U$

\begin{tabular}{llllll}
\hline$R(U \times U)$ & $x_{1}$ & $x_{2}$ & $x_{3}$ & $x_{4}$ & $x_{5}$ \\
\hline$x_{1}$ & $\langle 1,0\rangle$ & $\langle 0.5,0.7\rangle$ & $\langle 0.6,0.7\rangle$ & $\langle 0.4,0.3\rangle$ & $\langle 0.5,0.3\rangle$ \\
$x_{2}$ & $\langle 0.5,0.7\rangle$ & $\langle 1,0\rangle$ & $\langle 0.5,0.4\rangle$ & $\langle 0.5,0.6\rangle$ & $\langle 0.6,0.4\rangle$ \\
$x_{3}$ & $\langle 0.6,0.7\rangle$ & $\langle 0.5,0.4\rangle$ & $\langle 1,0\rangle$ & $\langle 0.7,0.6\rangle$ & $\langle 0.6,0.5\rangle$ \\
$x_{4}$ & $\langle 0.4,0.3\rangle$ & $\langle 0.5,0.6\rangle$ & $\langle 0.7,0.6\rangle$ & $\langle 1,0\rangle$ & $\langle 0.4,0.3\rangle$ \\
$x_{5}$ & $\langle 0.5,0.3\rangle$ & $\langle 0.6,0.4\rangle$ & $\langle 0.6,0.5\rangle$ & $\langle 0.4,0.3\rangle$ & $\langle 1,0\rangle$ \\
\hline
\end{tabular}

Table 2 The Pythagorean fuzzy relation on $U \times X$

\begin{tabular}{llllll}
\hline$R(U \times X)$ & $x_{1}$ & $x_{2}$ & $x_{3}$ & $x_{4}$ & $x_{5}$ \\
\hline$x_{1}$ & $\langle 0,1\rangle$ & $\langle 0,1\rangle$ & $\langle 0.6,0.7\rangle$ & $\langle 0,1\rangle$ & $\langle 0.5,0.3\rangle$ \\
$x_{2}$ & $\langle 0,1\rangle$ & $\langle 0,1\rangle$ & $\langle 0.5,0.4\rangle$ & $\langle 0,1\rangle$ & $\langle 0.6,0.4\rangle$ \\
$x_{3}$ & $\langle 0,1\rangle$ & $\langle 0,1\rangle$ & $\langle 1,0\rangle$ & $\langle 0,1\rangle$ & $\langle 0.6,0.5\rangle$ \\
$x_{4}$ & $\langle 0,1\rangle$ & $\langle 0,1\rangle$ & $\langle 0.7,0.6\rangle$ & $\langle 0,1\rangle$ & $\langle 0.4,0.3\rangle$ \\
$x_{5}$ & $\langle 0,1\rangle$ & $\langle 0,1\rangle$ & $\langle 0.6,0.5\rangle$ & $\langle 0,1\rangle$ & $\langle 1,0\rangle$ \\
\hline
\end{tabular}

$I(A, B)=\frac{\sum_{x \in U}\left(\left(\left(\mu_{A}^{2}(x) \wedge \mu_{B}^{2}(x)\right)+v_{A}^{2}(x)\right)\right.}{\sum_{x \in U}\left(\mu_{A}^{2}(x)+\left(v_{A}^{2}(x) \vee v_{B}^{2}(x)\right)\right)}$.

The inclusion measure $I(A, B)$ defined in Eq. 5 satisfies the four conditions of the Definition 4.

For example, two PFSs $A=\left\{\left\langle x_{1}, 0.9,0.3\right\rangle,\left\langle x_{2}, 0.4,0.7\right\rangle\right.$, $\left.\left\langle x_{3}, 0.8,0.4\right\rangle,\left\langle x_{4}, 0.7,0.2\right\rangle\right\}$ and $B=\left\{\left\langle x_{1}, 0.7,0.6\right\rangle,\left\langle x_{2}\right.\right.$, $\left.0.9,0.2\rangle,\left\langle x_{3}, 0.7,0.5\right\rangle,\left\langle x_{4}, 0.8,0.2\right\rangle\right\}$. Then $I(A, B)=$ $\frac{0.49+0.16+0.49+0.49+0.09+0.49+0.16+0.04}{0.81+0.16+0.64+0.49+0.36+0.49+0.25+0.04}=\frac{2.41}{3.24}=0.74382716$

\section{MG-PF-DTRSs based on inclusion measure}

In this section first we propose and study the models of inclusion measure-based MG-PF-DTRSs, within the framework of multi-granulation Pythagorean fuzzy approximation space.

Definition 6 Let $U$ be a finite universe. For any $X \subseteq U$ and $R_{k}(U \times X)(1 \leq k \leq m)$ be $m$ Pythagorean fuzzy relations on $U \times X$. Then, we call $\left(U, X, R_{k}(U \times X)(1 \leq k \leq m)\right)$ a multi-granulation Pythagorean fuzzy approximation space on $U$.

Definition 7 Let $R_{k}(U \times X)(1 \leq k \leq m)$ be $m$ Pythagorean fuzzy relations on $U \times X$, where $X \subseteq U$. For each $x \in U$, two PFSs $[x]_{\cap_{k=1}^{m}} R_{k}(U \times X)$ and $[x]_{\cup_{k=1}^{m}}^{m} R_{k}(U \times X)$ are defined as follows:

$$
\begin{gathered}
{[x]_{\cap_{k=1}^{m} R_{k}(U \times X)}=\left\{\left\langley, \wedge_{k=1}^{m} \mu_{R_{k}(U \times X)}(x, y),\right.\right.} \\
\left.\left.\vee_{k=1}^{m} v_{R_{k}(U \times X)}(x, y)\right\rangle \mid x \in U, y \in X\right\}
\end{gathered}
$$

and

$$
\begin{gathered}
{[x]_{\cup_{k=1}^{m} R_{k}(U \times X)}=\left\{\left\langley, \vee_{k=1}^{m} \mu_{R_{k}(U \times X)}(x, y),\right.\right.} \\
\left.\left.\wedge_{k=1}^{m} v_{R_{k}(U \times X)}(x, y)\right\rangle \mid x \in U, y \in X\right\}
\end{gathered}
$$

for all $y \in X$.

We are now ready to propose a model of four types of MG-PF-DTRSs. This model is shown by Fig. 1. The first two models, called Type-I multi-granulation Pythagorean fuzzy decision-theoretic rough set (Type-I MG-PF-DTRS) and Type-II multi-granulation Pythagorean fuzzy decisiontheoretic rough set (Type-II MG-PF-DTRS), are a class based on combination of relation first and then construction of approximations, as demonstrated in the upper half Fig. 1. The next two models, called Type-III multi-granulation Pythagorean fuzzy decision-theoretic rough set (Type-III MG-PF-DTRS) and multi-granulation Pythagorean fuzzy decision-theoretic rough set (Type-IV MG-PF-DTRS), are another class that is used the reverse order, as demonstrated in the lower half Fig. 1.

Now in the following we study four types PF-DTRSs based on the inclusion measure, within the framework of multi-granulation Pythagorean fuzzy approximation space, i.e., four types of MG-PF-DTRSs.

\section{Type-I MG-PF-DTRSs}

Definition 8 Let $\left(U, X, R_{k}(U \times X)(1 \leq k \leq m)\right)$ be a multigranulation Pythagorean fuzzy approximation space, for any $X \subseteq U$ with non-empty and finite universe of discourse $U$ and $m$ Pythagorean fuzzy relations $R_{k}(U \times X)(1 \leq k \leq m)$. For any $A(X)$ and $0 \leq \beta<\alpha \leq 1$, the inclusion measurebased Type-I multi-granulation Pythagorean fuzzy $\alpha$-lower and $\beta$-upper approximations of $A(X)$ w.r.t. ( $U, X, R_{k}(U \times$ $X)(1 \leq k \leq m))$ are defined, respectively, as follows:

$$
\begin{aligned}
& \operatorname{Apr}_{\cap_{k=1}^{m} R_{k}(U \times X)}^{\alpha}(A(X)) \\
& \quad=\left\{x \in U: I\left([x]_{\cap_{k=1}^{m} R_{k}(U \times X)}, A(X)\right) \geq \alpha\right\},
\end{aligned}
$$




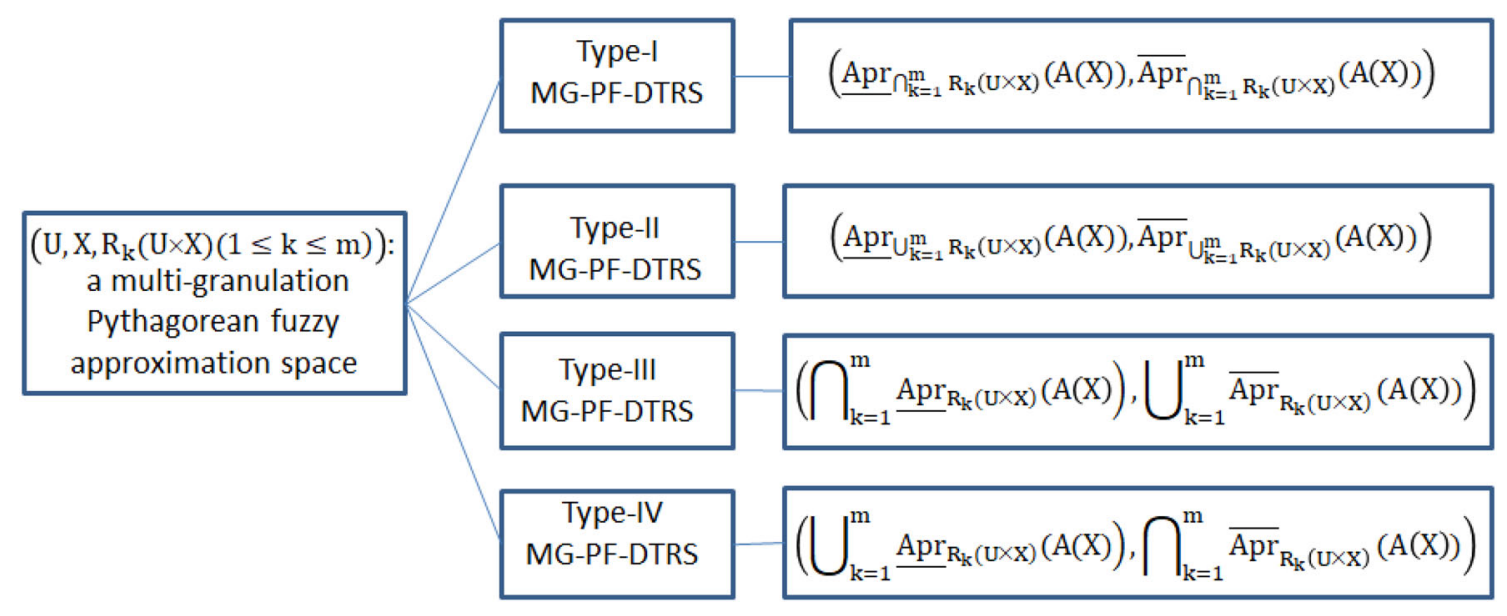

Fig. 1 Models of multi-granulation Pythagorean fuzzy rough sets

$$
\begin{aligned}
& \overline{\operatorname{Apr}}_{\cap_{k=1}^{m} R_{k}(U \times X)}^{\beta}(A(X)) \\
& \quad=\left\{x \in U: I\left([x]_{\cap_{k=1}^{m} R_{k}(U \times X)}, A(X)\right)>\beta\right\} .
\end{aligned}
$$

We call the pair $\left(\underline{\operatorname{Apr}}_{\cap_{k=1}^{\alpha} R_{k}(U \times X)}^{\alpha}(A(X)), \overline{\operatorname{Apr}}_{\cap_{k=1}^{m} R_{k}(U \times X)}^{\beta}\right.$ $(A(X)))$ as inclusion measure based Type-I $(\alpha, \beta)$-MG-PFDTRS of $A(X)$ w.r.t. $\left(U, X, R_{k}(U \times X)(1 \leq k \leq m)\right)$. The positive, negative and boundary regions of $A(X)$ w.r.t. $\left(U, R_{k}(1 \leq k \leq m)\right)$ are defined, respectively, as follows:

$$
\begin{aligned}
\operatorname{POS}^{\alpha}(A(X))= & \operatorname{Apr}_{\cap_{k=1}^{\alpha} R_{k}(U \times X)}^{\alpha}(A(X)), \\
\operatorname{NEG}^{\beta}(A(X))= & \left(\overline{\operatorname{Apr}}_{\cap_{k=1}^{m} R_{k}(U \times X)}^{\beta}(A(X))\right)^{c}, \\
\operatorname{BND}^{(\alpha, \beta)}(A(X))= & \overline{\operatorname{Apr}}_{\cap_{k=1}^{m} R_{k}(U \times X)}^{\beta}(A(X)) \\
& -\underline{\operatorname{Apr}}_{\cap_{k=1}^{m} R_{k}(U \times X)}^{\alpha}(A(X)) .
\end{aligned}
$$

Remark 1 For any $X=\left\{y_{1}, y_{2}, \ldots, y_{n}\right\} \subseteq U$ and for all $x \in U$, we have Eq. (10) from Eq. (7). and

$$
\overline{\operatorname{Apr}}_{\cap_{k=1}^{m} R_{k}(U \times X)}^{\beta_{2}}(A(X)) \subseteq \overline{\operatorname{Apr}}_{\cap_{k=1}^{m} R_{k}(U \times X)}^{\beta_{1}}(A(X))
$$

4. For any $X, Y \subseteq U$ with $A(X) \subseteq A(Y)$, we obtain

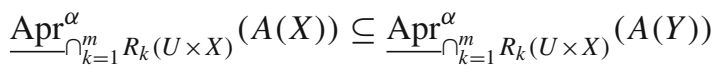

and

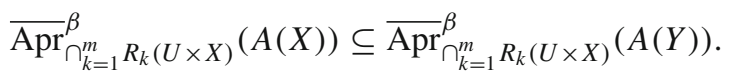

Remark 3 In the Definition 8, the Type-I $(\alpha, \beta)$-MG-PFDTRS is also refereed to as asymmetric MG-PF-DTRS, the aim of which is to approximate Pythagorean fuzzy concepts with high desired level of prediction accuracy. From Definition 8, we can derive the two special kinds of MG-PF-DTRSs are given, respectively, as follows:

$I\left([x]_{\cap_{k=1}^{m} R_{k}(U \times X)}, A(X)\right)=\frac{\sum_{i=1}^{n}\left(\left(\left(\wedge_{k=1}^{m} \mu_{R_{k}(U \times X)}^{2}\left(x, y_{i}\right)\right) \wedge \mu_{A(X)}^{2}\left(y_{i}\right)\right)+\left(\vee_{k=1}^{m} v_{R_{k}(U \times X)}^{2}\left(x, y_{i}\right)\right)\right)}{\sum_{i=1}^{n}\left(\left(\wedge_{k=1}^{m} \mu_{R_{k}(U \times X)}^{2}\left(x, y_{i}\right)\right)+\left(\left(\vee_{k=1}^{m} v_{R_{k}(U \times X)}^{2}\left(x, y_{i}\right)\right) \vee v_{A(X)}^{2}\left(y_{i}\right)\right)\right)}$

Remark 2 1. For any $X \subseteq U$, we obtain

$$
\operatorname{Apr}_{\cap_{k=1}^{m} R_{k}(U \times X)}^{\alpha}(A(X)) \subseteq \overline{\operatorname{Apr}}_{\cap_{k=1}^{m} R_{k}(U \times X)}^{\beta}(A(X)) .
$$

2. $\operatorname{Apr}_{\cap_{k=1}^{m} R_{k}(U \times U)}^{\alpha}(U)=U \overline{\operatorname{Apr}}_{\cap_{k=1}^{m} R_{k}(U \times U)}^{\beta}(U)$.

3. For any $X \subseteq U$ and $0 \leq \beta_{1} \leq \beta_{2} \leq \alpha_{1} \leq \alpha_{2} \leq 1$, we obtain

$$
\operatorname{Apr}_{\bigcap_{k=1}^{\alpha_{2}} R_{k}(U \times X)}(A(X)) \subseteq \operatorname{Apr}_{\bigcap_{k=1}^{\alpha_{1}} R_{k}(U \times X)}(A(X)) .
$$

1. The Type-I $\alpha$-MG-PF-DTRS (with $0.5<\alpha \leq 1$ ) of $A(X)$ w.r.t. $\left(U, X, R_{k}(U \times X)(1 \leq k \leq m)\right)$ is defined for any $X \subseteq U$ in terms of inclusion measure based Type-I multi-granulation Pythagorean fuzzy $\alpha$-lower and $\alpha$-upper approximations as

$$
\begin{aligned}
& \operatorname{Apr}_{\cap_{k=1}^{m} R_{k}(U \times X)}^{\alpha}(A(X)) \\
& \quad=\left\{x \in U: I\left([x]_{\cap_{k=1}^{m} R_{k}(U \times X)}, A(X)\right) \geq \alpha\right\},
\end{aligned}
$$




$$
\begin{aligned}
& \overline{\operatorname{Apr}}_{\cap_{k=1}^{m} R_{k}(U \times X)}^{\beta}(A(X)) \\
& \quad=\left\{x \in U: I\left([x]_{\cap_{k=1}^{m} R_{k}(U \times X)}, A(X)\right)>1-\alpha\right\} .
\end{aligned}
$$

$$
\begin{aligned}
\operatorname{BND}^{(\alpha, \beta)}(A(X))= & \overline{\operatorname{Apr}}_{\cup_{k=1}^{m} R_{k}(U \times X)}^{\beta}(A(X)) \\
& -\underline{\operatorname{Apr}}_{\cup_{k=1}^{m} R_{k}(U \times X)}(A(X)) .
\end{aligned}
$$

2. The Type-I 0.5-MG-PF-DTRS of $A(X)$ w.r.t. ( $U, X$, $\left.R_{k}(U \times X)(1 \leq k \leq m)\right)$ is defined for any $X \subseteq$
Remark 4 For any $X=\left\{y_{1}, y_{2}, \ldots, y_{n}\right\} \subseteq U$ and for all $x \in U$, we have Eq. (11) from Eq. (7).

$I\left([x]_{\cup_{k=1}^{m} R_{k}(U \times X)}, A(X)\right)=\frac{\sum_{i=1}^{n}\left(\left(\left(\vee_{k=1}^{m} \mu_{R_{k}(U \times X)}^{2}\left(x, y_{i}\right)\right) \wedge \mu_{A(X)}^{2}\left(y_{i}\right)\right)+\left(\wedge_{k=1}^{m} v_{R_{k}(U \times X)}^{2}\left(x, y_{i}\right)\right)\right)}{\sum_{i=1}^{n}\left(\left(\vee_{k=1}^{m} \mu_{R_{k}(U \times X)}^{2}\left(x, y_{i}\right)\right)+\left(\left(\wedge_{k=1}^{m} v_{R_{k}(U \times X)}^{2}\left(x, y_{i}\right)\right) \vee v_{A(X)}^{2}\left(y_{i}\right)\right)\right)}$,

$U$ in terms of inclusion measure based Type-I multigranulation Pythagorean fuzzy 0.5-lower and 0.5-upper approximations as

$$
\begin{aligned}
& \operatorname{Apr}_{\cap_{k=1}^{m} R_{k}(U \times X)}^{\alpha}(A(X)) \\
& \quad=\left\{x \in U: I\left([x]_{\cap_{k=1}^{m} R_{k}(U \times X)}, A(X)\right)>0.5\right\}, \\
& \overline{\operatorname{Apr}}_{\cap_{k=1}^{m} R_{k}(U \times X)}^{\beta}(A(X)) \\
& \quad=\left\{x \in U: I\left([x]_{\cap_{k=1}^{m} R_{k}(U \times X)}, A(X)\right) \geq 0.5\right\} .
\end{aligned}
$$

\section{Type-II MG-PF-DTRSs}

Definition 9 Let $\left(U, X, R_{k}(U \times X)(1 \leq k \leq m)\right)$ be a multigranulation Pythagorean fuzzy approximation space, for any $X \subseteq U$ with non-empty and finite universe of discourse $U$ and $m$ Pythagorean fuzzy relations $R_{k}(U \times X)(1 \leq k \leq m)$. For any $A(X)$ and $0 \leq \beta<\alpha \leq 1$, the inclusion measurebased Type-II multi-granulation Pythagorean fuzzy $\alpha$-lower and $\beta$-upper approximations of $A(X)$ w.r.t. $\left(U, X, R_{k}(U \times\right.$ $X)(1 \leq k \leq m))$ are defined, respectively, as follows:

$$
\begin{aligned}
& \underline{A p r}_{\cup_{k=1}^{m} R_{k}(U \times X)}^{\alpha}(A(X)) \\
& \quad=\left\{x \in U: I\left([x]_{\cup_{k=1}^{m} R_{k}(U \times X)}, A(X)\right) \geq \alpha\right\}, \\
& \overline{\operatorname{Apr}}_{\cup_{k=1}^{m} R_{k}(U \times X)}^{\beta}(A(X)) \\
& \quad=\left\{x \in U: I\left([x]_{\cup_{k=1}^{m} R_{k}(U \times X)}, A(X)\right)>\beta\right\} .
\end{aligned}
$$

We call the pair $\left(\underline{\operatorname{Apr}}_{\cup_{k=1}^{m} R_{k}(U \times X)}^{\alpha}(A(X))\right), \overline{A p r}_{\cup_{k=1}^{m} R_{k}(U \times X)}^{\beta}$ $(A(X))$ as inclusion measure-based Type-II $(\alpha, \beta)$-MG-PFDTRS of $A(X)$ w.r.t. $\left(U, X, R_{k}(U \times X)(1 \leq k \leq m)\right)$. The positive, negative and boundary regions of $A(X)$ w.r.t. $\left(U, X, R_{k}(U \times X)(1 \leq k \leq m)\right)$ are defined, respectively, as follows:

$\operatorname{POS}^{\alpha}(A(X))=\underline{\operatorname{Apr}}_{\cup_{k=1}^{m} R_{k}(U \times X)}^{\alpha}(A(X))$,
$\operatorname{NEG}^{\beta}(A(X))=\left(\overline{\operatorname{Apr}}_{\cup_{k=1}^{m} R_{k}(U \times X)}^{\beta}(A(X))\right)^{c}$,
Remark 51 . For any $X \subseteq U$, we obtain

$$
\operatorname{Apr}_{\cup_{k=1}^{m} R_{k}(U \times X)}^{\alpha}(A(X)) \subseteq \overline{\operatorname{Apr}}_{\cup_{k=1}^{m} R_{k}(U \times X)}^{\beta}(A(X))
$$

2. $\operatorname{Apr}_{\cup_{k=1}^{m} R_{k}(U \times U)}^{\alpha}(U)=U=\overline{\operatorname{Apr}}_{\cup_{k=1}^{m} R_{k}(U \times U)}^{\beta}(U)$.

3. For any $X \subseteq U$ and $0 \leq \beta_{1} \leq \beta_{2} \leq \alpha_{1} \leq \alpha_{2} \leq 1$, we obtain

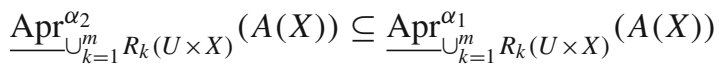

and

$$
\overline{\operatorname{Apr}}_{\cup_{k=1}^{m} R_{k}(U \times X)}^{\beta_{2}}(A(X)) \subseteq \overline{\operatorname{Apr}}_{\cup_{k=1}^{m} R_{k}}^{\beta_{1}}(A(X))
$$

4. For any $X, Y \subseteq U$ with $A(X) \subseteq A(Y)$, we obtain

$\operatorname{Apr}_{\cup_{k=1}^{\alpha} R_{k}(U \times X)}^{\alpha}(A(X)) \subseteq \underline{A p r}_{\cup_{k=1}^{\alpha} R_{k}(U \times X)}^{\alpha}(A(Y))$

and

$$
\overline{\operatorname{Apr}}_{\cup_{k=1}^{m} R_{k}(U \times X)}^{\beta}(A(X)) \subseteq \overline{\operatorname{Apr}}_{\cup_{k=1}^{m} R_{k}(U \times X)}^{\beta}(A(Y)) .
$$

Remark 6 From the Definition 9, we can derive the two special kinds of MG-PF-RSs, given, respectively, as follows:

1. The Type-II $\alpha$-MG-PF-DTRS (with $0.5<\alpha \leq 1$ ) of $A(X)$ w.r.t. $\left(U, X, R_{k}(U \times X)(1 \leq k \leq m)\right)$ is defined for any $X \subseteq U$ in terms of inclusion measure-based Type-II multi-granulation Pythagorean fuzzy $\alpha$-lower and $\alpha$-upper approximations as

$$
\begin{aligned}
& \operatorname{Apr}_{\cup_{k=1}^{m} R_{k}(U \times X)}^{\alpha}(A(X)) \\
& \quad=\left\{x \in U: I\left([x]_{\cup_{k=1}^{m} R_{k}(U \times X)}, A(X)\right) \geq \alpha\right\}, \\
& \overline{\operatorname{Apr}}_{\cup_{k=1}^{m} R_{k}(U \times X)}^{\beta}(A(X)) \\
& \quad=\left\{x \in U: I\left([x]_{\cup_{k=1}^{m} R_{k}(U \times X)}, A(X)\right)>1-\alpha\right\} .
\end{aligned}
$$


2. The Type-II 0.5-MG-PF-DTRS of $A(X)$ w.r.t. ( $U, X, R_{k}$ $(U \times X)(1 \leq k \leq m))$ is defined for any $X \subseteq U$ in terms of inclusion measure-based Type-II multi-granulation Pythagorean fuzzy 0.5-lower and 0.5-upper approximations as

$$
\begin{aligned}
\operatorname{BND}^{(\alpha, \beta)}(A(X))= & \cup_{k=1}^{m} \overline{A p r}_{R_{k}(U \times X)}^{\beta}(A(X)) \\
& -\cap_{k=1}^{m} \underline{\operatorname{Apr}}_{R_{k}(U \times X)}^{\alpha}(A(X)) .
\end{aligned}
$$

Remark 7 For any $X=\left\{y_{1}, y_{2}, \ldots, y_{n}\right\} \subseteq U$ and for all $x \in U$, we have Eqs. (12) and (13) from Eq. (7).

$$
\begin{aligned}
& \cap_{k=1}^{m} I\left([x]_{R_{k}(U \times X)}, A(X)\right)=\bigwedge_{k=1}^{m} \frac{\sum_{i=1}^{n}\left(\left(\mu_{R_{k}(U \times X)}^{2}\left(x, y_{i}\right) \wedge \mu_{A(X)}^{2}\left(y_{i}\right)\right)+v_{R_{k}(U \times X)}^{2}\left(x, y_{i}\right)\right)}{\sum_{i=1}^{n}\left(\mu_{R_{k}(U \times X)}^{2}\left(x, y_{i}\right)+\left(v_{R_{k}(U \times X)}^{2}\left(x, y_{i}\right) \vee v_{A(X)}^{2}\left(y_{i}\right)\right)\right.}, \\
& \cup_{k=1}^{m} I\left([x]_{R_{k}(U \times X)}, A(X)\right)=\bigvee_{k=1}^{m} \frac{\sum_{i=1}^{n}\left(\left(\mu_{R_{k}(U \times X)}^{2}\left(x, y_{i}\right) \wedge \mu_{A(X)}^{2}\left(y_{i}\right)\right)+v_{R_{k}(U \times X)}^{2}\left(x, y_{i}\right)\right)}{\sum_{i=1}^{n}\left(\mu_{R_{k}(U \times X)}^{2}\left(x, y_{i}\right)+\left(v_{R_{k}(U \times X)}^{2}\left(x, y_{i}\right) \vee v_{A(X)}^{2}\left(y_{i}\right)\right)\right.}
\end{aligned}
$$

$$
\begin{aligned}
& \underline{\operatorname{Apr}^{\alpha}} \cup_{k=1}^{\alpha} R_{k}(U \times X) \\
& \quad=\left\{x \in U: I\left([x] \cup_{k=1}^{m} R_{k}(U \times X), A(X)\right)>0.5\right\}, \\
& \quad \overline{\operatorname{Apr}}_{\cup_{k=1}^{m} R_{k}(U \times X)}^{\beta}(A(X)) \\
& \quad=\left\{x \in U: I\left([x]_{\cup_{k=1}^{m} R_{k}(U \times X)}, A(X)\right) \geq 0.5\right\} .
\end{aligned}
$$

\section{Type-III MG-PF-DTRSs}

Definition 10 Let $\left(U, X, R_{k}(U \times X)(1 \leq k \leq m)\right)$ be a multi-granulation Pythagorean fuzzy approximation space, for any $X \subseteq U$ with non-empty and finite universe of discourse $U$ and $m$ Pythagorean fuzzy relations $R_{k}(U \times X)(1 \leq$ $k \leq m)$. For any $A(X)$ and $0 \leq \beta<\alpha \leq 1$, the inclusion measure-based Type-III multi-granulation Pythagorean fuzzy $\alpha$-lower and $\beta$-upper approximations of $A(X)$ w.r.t. $\left(U, X, R_{k}(U \times X)(1 \leq k \leq m)\right)$ are defined, respectively, as follows:

$$
\begin{aligned}
& \cap_{k=1}^{m} \operatorname{Apr}_{R_{k}(U \times X)}^{\alpha}(A(X)) \\
& \quad=\left\{x \in U: \cap_{k=1}^{m} I\left([x]_{R_{k}(U \times X)}, A(X)\right) \geq \alpha\right\}, \\
& \cup_{k=1}^{m} \overline{\operatorname{Apr}}_{R_{k}(U \times X)}^{\beta}(A(X)) \\
& \quad=\left\{x \in U: \cup_{k=1}^{m} I\left([x]_{R_{k}(U \times X)}, A(X)\right)>\beta\right\} .
\end{aligned}
$$

We call the pair $\left(\cap_{k=1}^{m} \underline{\operatorname{Apr}}_{R_{k}(U \times X)}^{\alpha}(A(X)), \cup_{k=1}^{m} \overline{\operatorname{Apr}}_{R_{k}(U \times X)}^{\beta}\right.$ $(A(X))$ as inclusion measure-based Type-III $(\alpha, \beta)$-MGPF-DTRS of $A$ w.r.t. $\left(U, X, R_{k}(U \times X)(1 \leq k \leq m)\right)$. The positive, negative and boundary regions of $A(X)$ w.r.t. $\left(U, X, R_{k}(U \times X)(1 \leq k \leq m)\right)$ are defined, respectively, as follows:

$$
\begin{aligned}
& \operatorname{POS}^{\alpha}(A(X))=\cap_{k=1}^{m} \operatorname{Apr}_{R_{k}(U \times X)}^{\alpha}(A(X)), \\
& \operatorname{NEG}^{\beta}(A(X))=\left(\cup_{k=1}^{m} \overline{\operatorname{Apr}}_{R_{k}(U \times X)}^{\beta}(A(X))\right)^{c},
\end{aligned}
$$

Remark 8 1. For any $X \subseteq U$, we obtain

$$
\cap_{k=1}^{m} \underline{\operatorname{Apr}}_{R_{k}(U \times X)}^{\alpha}(A(X)) \subseteq \cup_{k=1}^{m} \overline{\operatorname{Apr}}_{R_{k}(U \times X)}^{\beta}(A(X)) .
$$

2. $\cap_{k=1}^{m} \operatorname{Apr}_{R_{k}(U \times U)}^{\alpha}(U)=U \cup_{k=1}^{m} \overline{\operatorname{Apr}}_{R_{k}(U \times U)}^{\beta}(U)$.

3. For any $X \subseteq U$ and $0 \leq \beta_{1} \leq \beta_{2} \leq \alpha_{1} \leq \alpha_{2} \leq 1$, we obtain

$$
\cap_{k=1}^{m} \underline{\operatorname{Apr}}_{R_{k}}^{\alpha_{2}}(A) \subseteq \cap_{k=1}^{m} \underline{\operatorname{Apr}}_{R_{k}(U \times X)}^{\alpha_{1}}(A(X))
$$

and

$$
\cup_{k=1}^{m} \overline{\operatorname{Apr}}_{R_{k}(U \times X)}^{\beta_{2}}(A(X)) \subseteq \cup_{k=1}^{m} \overline{\operatorname{Apr}}_{R_{k}(U \times X)}^{\beta_{1}}(A(X)) .
$$

4. For any $X, Y \subseteq U$ with $A(X) \subseteq B(X)$, we obtain

$$
\cap_{k=1}^{m} \operatorname{Apr}_{R_{k}(U \times X)}^{\alpha}(A(X)) \subseteq \cap_{k=1}^{m} \operatorname{Apr}_{R_{k}(U \times X)}^{\alpha}(A(Y))
$$

and

$$
\cup_{k=1}^{m} \overline{\operatorname{Apr}}_{R_{k}(U \times X)}^{\beta}(A(X)) \subseteq \cup_{k=1}^{m} \overline{\operatorname{Apr}}_{R_{k}(U \times X)}^{\beta}(A(Y)) .
$$

Remark 9 From the Definition 10, we can derive the two special kinds of MG-PF-RSs, are given, respectively, as follows:

1. the Type-III $\alpha$-MG-PF-DTRS (with $0.5<\alpha \leq 1$ ) of $A(X)$ w.r.t. $\left(U, X, R_{k}(U \times X)(1 \leq k \leq m)\right)$ is defined for any $X \subseteq U$ in terms of inclusion measure-based Type-III multi-granulation Pythagorean fuzzy $\alpha$-lower and $\alpha$-upper approximations as

$$
\begin{aligned}
& \cap_{k=1}^{m} \operatorname{Apr}_{R_{k}(U \times X)}^{\alpha}(A(X)) \\
& \quad=\left\{x \in U: \cap_{k=1}^{m} I\left([x]_{R_{k}(U \times X)}, A(X)\right) \geq \alpha\right\}, \\
& \cup_{k=1}^{m} \overline{\operatorname{Apr}}_{R_{k}(U \times X)}^{\beta}(A(X)) \\
& \quad=\left\{x \in U: \cup_{k=1}^{m} I\left([x]_{R_{k}(U \times X)}, A(X)\right)>1-\alpha\right\} .
\end{aligned}
$$


2. the Type-III 0.5-MG-PF-DTRS of $A(X)$ w.r.t. ( $U, X, R_{k}$ $(U \times X)(1 \leq k \leq m))$ is defined for any $X \subseteq U$ in terms of inclusion measure-based Type-III multi-granulation Pythagorean fuzzy 0.5-lower and 0.5-upper approximations as

$$
\begin{aligned}
& \cap_{k=1}^{m} \operatorname{Apr}_{R_{k}(U \times X)}^{\alpha}(A(X)) \\
& \quad=\left\{x \in U: \cap_{k=1}^{m} I\left([x]_{R_{k}(U \times X)}, A(X)\right)>0.5\right\}, \\
& \cup_{k=1}^{m} \overline{\operatorname{Apr}}_{R_{k}(U \times X)}^{\beta}(A(X)) \\
& \quad=\left\{x \in U: \cup_{k=1}^{m} I\left([x]_{R_{k}(U \times X)}, A(X)\right) \geq 0.5\right\} .
\end{aligned}
$$

\section{Type-IV MG-PF-DTRSs}

Definition 11 Let $\left(U, X, R_{k}(U \times X)(1 \leq k \leq m)\right)$ be a multi-granulation Pythagorean fuzzy approximation space, for any $X \subseteq U$ with non-empty and finite universe of discourse $U$ and $m$ Pythagorean fuzzy relations $R_{k}(U \times X)(1 \leq$ $k \leq m)$. For any $A(X)$ and $0 \leq \beta<\alpha \leq 1$, the inclusion measure-based Type-IV multi-granulation Pythagorean fuzzy $\alpha$-lower and $\beta$-upper approximations of $A(X)$ w.r.t. $\left(U, X, R_{k}(U \times X)(1 \leq k \leq m)\right)$ are defined, respectively, as follows:

$$
\begin{aligned}
& \cup_{k=1}^{m}{ }_{\operatorname{Apr}^{\alpha}}^{\alpha} R_{k}(U \times X) \\
& \quad=\left\{x \in U: \cup_{k=1}^{m} I\left([x]_{R_{k}(U \times X)}, A(X)\right) \geq \alpha\right\}, \\
& \cap_{k=1}^{m} \overline{\operatorname{Apr}}_{R_{k}(U \times X)}^{\beta}(A(X)) \\
& \quad=\left\{x \in U: \cap_{k=1}^{m} I\left([x]_{R_{k}(U \times X)}, A(X)\right)>\beta\right\} .
\end{aligned}
$$

We call the pair $\left(\cup_{k=1}^{m} \underline{\operatorname{Apr}}_{R_{k}(U \times X)}^{\alpha}(A(X)), \cap_{k=1}^{m} \overline{\operatorname{Apr}}_{R_{k}(U \times X)}^{\beta}\right.$ $(A(X))$ as inclusion measure-based Type-IV $(\alpha, \beta)$-MG-PFDTRS of $A(X)$ w.r.t. $\left(U, X, R_{k}(U \times X)(1 \leq k \leq m)\right)$. The positive, negative and boundary regions of $A(X)$ w.r.t. $\left(U, X, R_{k}(U \times X)(1 \leq k \leq m)\right)$ are defined, respectively, as follows:

$$
\begin{aligned}
& \operatorname{POS}^{\alpha}(A(X))=\cup_{k=1}^{m} \underline{\operatorname{Apr}}_{R_{k}(U \times X)}^{\alpha}(A(X)), \\
& \mathrm{NEG}^{\beta}(A(X))=\left(\cap_{k=1}^{m} \overline{\operatorname{Apr}}_{R_{k}(U \times X)}^{\beta}(A(X))\right)^{c}, \\
& \operatorname{BND}^{(\alpha, \beta)}(A(X))=\cap_{k=1}^{m} \overline{\operatorname{Apr}}_{R_{k}(U \times X)}^{\beta}(A(X)) \\
& -\cup_{k=1}^{m} \operatorname{Apr}_{R_{k}(U \times X)}^{\alpha}(A(X)) \text {. }
\end{aligned}
$$

Remark 10 1. $\cup_{k=1}^{m} \underline{\operatorname{Apr}}_{R_{k}(U \times U)}^{\alpha}(U)=U \cap_{k=1}^{m} \overline{\operatorname{Apr}}_{R_{k}(U \times U)}^{\beta}$ $(U)$.

2. For any $X \subseteq U$ and $0 \leq \beta_{1} \leq \beta_{2} \leq \alpha_{1} \leq \alpha_{2} \leq 1$, we obtain

$$
\cup_{k=1}^{m} \underline{\operatorname{Apr}}_{R_{k}(U \times X)}^{\alpha_{2}}(A(X)) \subseteq \cup_{k=1}^{m} \underline{\operatorname{Apr}}_{R_{k}(U \times X)}^{\alpha_{1}}(A(X))
$$

and

$$
\cap_{k=1}^{m} \overline{\operatorname{Apr}}_{R_{k}(U \times X)}^{\beta_{2}}(A(X)) \subseteq \cap_{k=1}^{m} \overline{\operatorname{Apr}}_{R_{k}(U \times X)}^{\beta_{1}}(A(X)) .
$$

3. For any $X, Y \subseteq U$ with $A(X) \subseteq A(Y)$, we obtain

$$
\cup_{k=1}^{m} \underline{\operatorname{Apr}}_{R_{k}(U \times X)}^{\alpha}(A(X)) \subseteq \cup_{k=1}^{m} \underline{\operatorname{Apr}}_{R_{k}(U \times X)}^{\alpha}(A(Y))
$$

and

$$
\cap_{k=1}^{m} \overline{\operatorname{Apr}}_{R_{k}(U \times X)}^{\beta}(A(X)) \subseteq \cap_{k=1}^{m} \overline{\operatorname{Apr}}_{R_{k}(U \times X)}^{\beta}(A(Y)) .
$$

Remark 11 From the Definition 11, we can derive the two special kinds of MG-PF-RSs, are given, respectively, as follows:

1. the Type-IV $\alpha$-MG-PF-DTRS (with $0.5<\alpha \leq 1$ ) of $A(X)$ w.r.t. $\left(U, X, R_{k}(U \times X)(1 \leq k \leq m)\right)$ is defined for any $X \subseteq U$ in terms of inclusion measure-based Type-IV multi-granulation Pythagorean fuzzy $\alpha$-lower and $\alpha$-upper approximations as

$$
\begin{aligned}
& \cup_{k=1}^{m} \operatorname{Apr}_{R_{k}(U \times X)}^{\alpha}(A(X)) \\
& \quad=\left\{x \in U: \cup_{k=1}^{m} I\left([x]_{R_{k}(U \times X)}, A(X)\right) \geq \alpha\right\}, \\
& \cap_{k=1}^{m} \overline{\operatorname{Apr}}_{R_{k}(U \times X)}^{\beta}(A(X)) \\
& \quad=\left\{x \in U: \cap_{k=1}^{m} I\left([x]_{R_{k}(U \times X)}, A(X)\right)>1-\alpha\right\} .
\end{aligned}
$$

2. the Type-IV 0.5-MG-PF-DTRS of $A(X)$ w.r.t. ( $U, X, R_{k}$ $(U \times X)(1 \leq k \leq m))$ is defined for any $X \subseteq U$ in terms of inclusion measure-based Type-IV multi-granulation Pythagorean fuzzy 0.5-lower and 0.5-upper approximations as

$$
\begin{aligned}
& \cup_{k=1}^{m} \operatorname{Apr}_{R_{k}(U \times X)}^{\alpha}(A(X)) \\
& \quad=\left\{x \in U: \cup_{k=1}^{m} I\left([x]_{R_{k}(U \times X)}, A(X)\right)>0.5\right\}, \\
& \cap_{k=1}^{m} \overline{\operatorname{Apr}}_{R_{k}(U \times X)}^{\beta}(A(X)) \\
& \quad=\left\{x \in U: \cap_{k=1}^{m} I\left([x]_{R_{k}(U \times X)}, A(X)\right) \geq 0.5\right\} .
\end{aligned}
$$

\section{Uncertainty measures}

In this section, several measures are utilized to calculate the uncertainty of these models which is discussed in previous section. The uncertainty of knowledge is caused by the boundary regions, in the view point of approximations. The larger the boundary area is, the more the uncertainty. The accuracy, roughness and approximation quality are studied in the next.

Definition 12 Let $\left(U, X, R_{k}(U \times X)(1 \leq k \leq m)\right)$ be a multi-granulation Pythagorean fuzzy approximation space, 
for any $X \subseteq U$ with non-empty and finite universe of discourse $U$ and $m$ Pythagorean fuzzy relations $R_{k}(U \times X)(1 \leq$ $k \leq m)$. For any $A(X)$ and $0 \leq \beta<\alpha \leq 1$, the Type-I MGPF-DTRS accuracy $\rho_{\text {I }}$, Type-II MG-PF-DTRS accuracy $\rho_{\text {II }}$, Type-III MG-PF-DTRS accuracy $\rho_{\text {III }}$ and Type-IV MG-PFDTRS accuracy $\rho_{\mathrm{IV}}$ of $A(X)$ w.r.t. $\left(U, X, R_{k}(U \times X)(1 \leq\right.$ $k \leq m)$ ) are defined, respectively, as follows:

$$
\begin{gathered}
\rho_{\mathrm{I}}=\frac{\mid \frac{\operatorname{Apr}_{\bigcap_{k=1}^{m} R_{k}(U \times X)}^{\alpha}(A(X)) \mid}{\left|\overline{\operatorname{Apr}}_{\bigcap_{k=1}^{m} R_{k}(U \times X)}^{\beta}(A(X))\right|},}{\rho_{\mathrm{II}}=\frac{\left|{\operatorname{\operatorname {Apr}^{\alpha }}}_{\cup_{k=1}^{m} R_{k}(U \times X)}(A(X))\right|}{\left|\overline{\operatorname{Apr}}_{\cup_{k=1}^{m} R_{k}(U \times X)}^{\beta}(A(X))\right|},} \\
\rho_{\mathrm{III}}=\frac{\left|\cap_{k=1}^{m} \underline{\operatorname{Apr}}_{R_{k}(U \times X)}^{\alpha}(A(X))\right|}{\left|\cup_{k=1}^{m} \overline{\operatorname{Apr}}_{R_{k}(U \times X)}^{\beta}(A(X))\right|}, \\
\rho_{\mathrm{IV}}=\frac{\left|\cup_{k=1}^{m} \overline{\operatorname{Apr}}_{R_{k}(U \times X)}^{\alpha}(A(X))\right|}{\left|\cap_{k=1}^{m} \overline{\operatorname{Apr}}_{R_{k}(U \times X)}^{\beta}(A(X))\right|} .
\end{gathered}
$$

Definition 13 Let $\left(U, X, R_{k}(U \times X)(1 \leq k \leq m)\right)$ be a multi-granulation Pythagorean fuzzy approximation space, for any $X \subseteq U$ with non-empty and finite universe of discourse $U$ and $m$ Pythagorean fuzzy relations $R_{k}(U \times X)(1 \leq$ $k \leq m)$. For any $A(X)$ and $0 \leq \beta<\alpha \leq 1$, the Type-I MGPF-DTRS approximation degree $\sigma_{\text {I }}$, Type-II MG-PF-DTRS approximation degree $\sigma_{\text {II }}$, Type-III MG-PF-DTRS approximation degree $\sigma_{\mathrm{III}}$ and Type-IV MG-PF-DTRS approximation degree $\sigma_{\mathrm{IV}}$ of $A(X)$ w.r.t. $\left(U, X, R_{k}(U \times X)(1 \leq k \leq\right.$ $m)$ ) are defined, respectively, as follows:

$$
\begin{aligned}
& \sigma_{\mathrm{I}}=\frac{\left|\operatorname{Apr}_{\bigcap_{k=1}^{\alpha} R_{k}(U \times X)}^{\alpha}(A(X))\right|}{|X|}, \\
& \sigma_{\mathrm{II}}=\frac{\left|\operatorname{Apr}_{\cup_{k=1}^{m} R_{k}(U \times X)}^{\alpha}(A(X))\right|}{|X|}, \\
& \sigma_{\mathrm{III}}=\frac{\left|\cap_{k=1}^{m} \operatorname{Apr}_{R_{k}(U \times X)}^{\alpha}(A(X))\right|}{|X|}, \\
& \sigma_{\mathrm{IV}}=\frac{\left|\cup_{k=1}^{m} \operatorname{Apr}_{R_{k}(U \times X)}^{\alpha}(A(X))\right|}{|X|} .
\end{aligned}
$$

Definition 14 Let $\left(U, X, R_{k}(U \times X)(1 \leq k \leq m)\right)$ be a multi-granulation Pythagorean fuzzy approximation space, for any $X \subseteq U$ with non-empty and finite universe of discourse $U$ and $m$ Pythagorean fuzzy relations $R_{k}(U \times X)(1 \leq$ $k \leq m)$. For any $A(X)$ and $0 \leq \beta<\alpha \leq 1$, the Type-I MGPF-DTRS approximation quality $\omega_{\text {I }}$, Type-II MG-PF-DTRS approximation quality $\omega_{\text {II }}$, Type-III MG-PF-DTRS approximation quality $\omega_{\text {III }}$ and Type-IV MG-PF-DTRS approximation quality $\omega_{\mathrm{IV}}$ of $A(X)$ w.r.t. $\left(U, X, R_{k}(U \times X)(1 \leq k \leq\right.$ $m)$ ) are defined, respectively, as follows:

$$
\begin{aligned}
& \omega_{\mathrm{I}}=\frac{\left|\mathrm{Apr}_{\bigcap_{k=1}^{m} R_{k}(U \times X)}^{\alpha}(A(X))\right|}{|U|},
\end{aligned}
$$

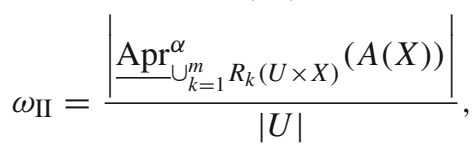

$$
\begin{aligned}
& \omega_{\text {III }}=\frac{\left|\cap_{k=1}^{m} \underline{A p r}_{R_{k}(U \times X)}^{\alpha}(A(X))\right|}{|U|}, \\
& \omega_{\mathrm{IV}}=\frac{\left|\cup_{k=1}^{m} \underline{\operatorname{Apr}}_{R_{k}(U \times X)}^{\alpha}(A(X))\right|}{|U|} .
\end{aligned}
$$

\section{Decision-making to incomplete multi-source information systems using MG-PF-DTRSs}

In this section, based on the MG-PF-DTRSs and their uncertainty measures established in "MG-PF-DTRSs based on inclusion measure" and "Uncertainty measures", we will construct a new method and approach to decision-making with incomplete multi-source information systems. Also, we will present the decision-making algorithm and the general steps for established method in detail.

\section{Incomplete multi-source information systems and the similarity degrees}

Definition 15 (Lin et al. [22]) A multi-source information system is MSIS $=\left\{\mathrm{IS}_{l} \mid \mathrm{IS}_{l}=\left(U, \mathrm{AT}_{l}, V=\right.\right.$ $\left.\left\{\left(V_{a}\right)_{a \in \mathrm{AT}}\right\}, f_{l}\right\}$, where

1. $U$ is a finite non-empty set of objects, called the universe;

2. $\mathrm{AT}_{l}$ is a non-empty finite set of attributes of each subsystem;

3. $\left\{V_{a}\right\}$ is the domain of the attribute $a \in \mathrm{AT}_{l}$; and

4. $f_{l}: U \times \mathrm{AT}_{l} \mapsto\left\{\left(V_{a}\right)_{a \in \mathrm{AT}_{l}}\right\}$ such that for all $x \in U$ and $a \in \mathrm{AT}_{l}, f(x, a) \in V_{a}$.

Definition 16 An incomplete multi-source information system (IMSIS) indicates the precise attribute values $V_{a}$ for some objects are unknown. In this paper, the IMSIS is still denoted without confusion by IMSIS $=\left\{\mathrm{IS}_{l} \mid \mathrm{IS}_{l}=\left(U, \mathrm{AT}_{l}, V, f_{l}\right\}\right.$. Here $V=\left\{\left(V_{a}\right)_{a \in \mathrm{AT}_{l}}\right\} \cup\{*\}$, the special symbol "*" is used to indicate the unknown value. For instance, if $f(x, a)=*$, the value of object $x$ is unknown on the attribute $a$. 
Liu et al. [23] handle the incomplete single source information system and compute the similarity degree between two objects. Their similarity degree between two objects is fuzzy set. Here, we handle the incomplete multi-source information system and compute the similarity degree between two objects. Our similarity degree between two objects is PFS.

Given an IMSIS $=\left\{\mathrm{IS}_{l} \mid \mathrm{IS}_{l}=\left(U, \mathrm{AT}_{l}, V, f_{l}\right\}\right.$, suppose the IMSIS contains $n$ objects and $t$ attributes, $U=$ $\left\{x_{1}, x_{2}, \ldots, x_{n}\right\}, \mathrm{AT}_{l} \subseteq\left\{a_{1}, a_{2}, \ldots, a_{t}\right\}$. For $\forall x, y \in U$, $\forall a_{i} \in \mathrm{AT}_{l}$, the relations between $\left(x, a_{i}\right)$ and $\left(y, a_{i}\right)$ can be treated as following four scenarios:

1. Consideration of $\left(x, a_{i}\right) \neq *$ and $\left(y, a_{i}\right) \neq *,\left(x, a_{i}\right)$ and $\left(y, a_{i}\right)$ are equality iff $\left(x, a_{i}\right)=\left(y, a_{i}\right)$;

2. Consideration of $\left(x, a_{i}\right) \neq *$ and $\left(y, a_{i}\right) \neq *,\left(x, a_{i}\right)$ and $\left(y, a_{i}\right)$ are not the same if $\left(x, a_{i}\right) \neq\left(y, a_{i}\right)$;

3. Consideration of $\left(x, a_{i}\right)=*$ or $\left(y, a_{i}\right)=*$, because of the unknown value "*" is treated as "do not care" conditions, it has the probability of $\frac{1}{V_{a_{i}}}$ to equal to one certain value of $V_{a_{i}}\left(V_{a_{i}}\right.$ is a domain of the attribute $a_{i}$, $\left|V_{a_{i}}\right|$ denotes the cardinality of $\left.a_{i}\right)$.

4. Consideration of $\left(x, a_{i}\right)=\left(y, a_{i}\right)=*$, both of $a_{i}(x)$ and $a_{i}(y)$ have the probability of $\frac{1}{\left|V_{a_{i}}\right|}$ to equal to one certain value of $V_{a_{i}}$, so the joint probability of $\left(x, a_{i}\right)=\left(y, a_{i}\right)$ is $\frac{1}{\left|V_{a_{i}}\right|^{2}}$.

With the above discussions, we apply the concepts of PFSs, the similarity degrees between $x$ and $y$ on $a_{i}$ can be written in the following way:

$$
\begin{aligned}
& \mu_{\operatorname{Sim}\left(a_{i}\right)}(x, y) \\
& \quad= \begin{cases}1 & \left(x, a_{i}\right)=\left(y, a_{i}\right) \neq * ; \\
0 & \left(x, a_{i}\right) \neq\left(y, a_{i}\right) \wedge\left(x, a_{i}\right) \neq * \wedge\left(y, a_{i}\right) \neq * ; \\
\frac{1}{\left|V_{a_{i}}\right|} & \left(x, a_{i}\right)=* \vee\left(y, a_{i}\right)=* ; \\
\frac{1}{\left|V_{a_{i}}\right|^{2}} & \left(x, a_{i}\right)=* \wedge\left(y, a_{i}\right)=*\end{cases}
\end{aligned}
$$

and

$$
\begin{aligned}
& v_{\operatorname{Sim}\left(a_{i}\right)}(x, y) \\
& = \begin{cases}0 & \left(x, a_{i}\right)=\left(y, a_{i}\right) \neq * ; \\
1 & \left(x, a_{i}\right) \neq\left(y, a_{i}\right) \wedge\left(x, a_{i}\right) \neq * \wedge\left(y, a_{i}\right) \neq * ; \\
\sqrt{1-\frac{1}{\mid V_{\left.a_{i}\right|^{2}}}} & \left(x, a_{i}\right)=* \vee\left(y, a_{i}\right)=* ; \\
\sqrt{1-\frac{1}{\left|V_{a_{i}}\right|^{4}}} & \left(x, a_{i}\right)=* \wedge\left(y, a_{i}\right)=*\end{cases}
\end{aligned}
$$

Table 3 A car incomplete multi-source information system

\begin{tabular}{llllll}
\hline$U$ & $\mathrm{EC}_{1}$ & & & $\mathrm{EC} 2$ & \\
\cline { 2 - 3 } \cline { 5 - 6 } & $c_{1}$ & $c_{3}$ & & $c_{2}$ & $c_{4}$ \\
\hline$x_{1}$ & High & Full & Low & Low \\
$x_{2}$ & Low & Medium & $*$ & Low \\
$x_{3}$ & $*$ & Compact & $*$ & Low \\
$x_{4}$ & High & Full & $*$ & High \\
$x_{5}$ & $*$ & Full & $*$ & High \\
$x_{6}$ & Low & Compact & High & $*$ \\
\hline
\end{tabular}

If $\mathrm{AT}_{l}=\left\{b_{1}, b_{1}, \ldots, b_{s}\right\} \subseteq\left\{a_{1}, a_{2}, \ldots, a_{t}\right\}$, then the similarity membership and non-membership degrees between $x$ and $y$ are calculated as follows:

$\begin{aligned} \mu_{R_{\mathrm{AT}_{l}}(U \times U)}(x, y) & =\sum_{i=1}^{s} \frac{\mu_{\operatorname{Sim}\left(b_{i}\right)}(x, y)}{s} \\ v_{R_{\mathrm{AT}_{l}}(U \times U)}(x, y) & =\sum_{i=1}^{s} \frac{v_{\operatorname{Sim}\left(b_{i}\right)}(x, y)}{s} .\end{aligned}$

Using Eqs. (28) and (29), from the Definition 3 we have:

$$
\begin{aligned}
R_{k}(U \times U)= & \left\{\left\langle(x, y), \sum_{i=1}^{s} \frac{\mu_{\operatorname{Sim}\left(b_{i}\right)}(x, y)}{s},\right.\right. \\
& \left.\left.\sum_{i=1}^{s} \frac{v_{\operatorname{Sim}\left(b_{i}\right)}(x, y)}{s}\right\rangle \mid x, y \in U\right\},
\end{aligned}
$$

where $1 \leq k \leq l$.

Example 1 Let us consider an evaluation problem of a car depicted by an IMSIS presented in Table 3. Suppose that $U=\left\{x_{1}, x_{2}, x_{3}, x_{4}, x_{5}, x_{6}\right\}$ is a set of six cars. Every car in each sub-information (source) system, denoted by $\mathrm{EC}_{1}$ and $\mathrm{EC}_{2}$, is described by two attributes. They are $c_{1}=$ Price, $c_{2}=$ Mileage, $c_{3}=$ Size, $c_{4}=$ Max-speed, respectively. The domains of the attributes are as follows: $V_{c_{1}}=\{$ High, Low $\}$, $V_{c_{2}}=$ \{High, Low $\}, V_{c_{3}}=$ Full, Medium, Compact $\}$, $V_{c_{4}}=\{$ High, Low $\}$.

According to Table 3 and Eqs. (26) and 27) we easily get $\mu_{\operatorname{Sim}\left(c_{1}\right)}\left(x_{1}, x_{2}\right)=0, \mu_{\operatorname{Sim}\left(c_{3}\right)}\left(x_{1}, x_{2}\right)=0, \nu_{\operatorname{Sim}\left(c_{2}\right)}\left(x_{1}, x_{2}\right)$ $=1$ and $v_{\operatorname{Sim}\left(c_{4}\right)}\left(x_{1}, x_{2}\right)=1$. From Eqs. (28) and (29) we have: $\mu_{R_{\mathrm{EC}_{1}}(U)}\left(x_{1}, x_{2}\right)=\frac{0+0}{2}=0$ and $v_{R_{\mathrm{EC}_{1}}(U)}\left(x_{1}, x_{2}\right)=$ $\frac{1+1}{2}=1$. Similarly, we compute $\mu_{R_{\mathrm{EC}_{1}}(U)}\left(x_{i}, x_{j}\right)$ and $v_{R_{\mathrm{EC}_{1}}(U)}\left(x_{i}, x_{j}\right)$ for all $x_{i}, x_{j} \in U(i, j=1,2, \ldots, 5)$, which is outlined in Table 4.

In the same way, we also compute $\mu_{R_{\mathrm{EC}_{2}}(U)}\left(x_{i}, x_{j}\right)$ and $v_{R_{\mathrm{EC}}(U)}\left(x_{i}, x_{j}\right)$ for all $x_{i}, x_{j} \in U(i, j=1,2, \ldots, 5)$ but here it is not necessary. 
Table 4 The computing results of $R_{\mathrm{EC}_{1}}(U)$

\begin{tabular}{|c|c|c|c|c|c|c|}
\hline$R_{\mathrm{EC}_{1}}(U \times U)$ & $x_{1}$ & $x_{2}$ & $x_{3}$ & $x_{4}$ & $x_{5}$ & $x_{6}$ \\
\hline$x_{1}$ & $\langle 1,0\rangle$ & $\langle 0,1\rangle$ & $\langle 0.2500,0.9330\rangle$ & $\langle 1,0\rangle$ & $\langle 0.7500,0.4303\rangle$ & $\langle 0,1\rangle$ \\
\hline$x_{2}$ & & $\langle 1,0\rangle$ & $\langle 0.2500,0.6330\rangle$ & $\langle 0,1\rangle$ & $\langle 0.2500,0.9330\rangle$ & $\langle 0.5000,0.5000\rangle$ \\
\hline$x_{3}$ & & & $\langle 1,0\rangle$ & $\langle 0.2500,0.9330\rangle$ & $\langle 0.1250,0.9841\rangle$ & $\langle 0.2500,0.9330\rangle$ \\
\hline$x_{4}$ & & & & $\langle 1,0\rangle$ & $\langle 0.7500,0.4330\rangle$ & $\langle 0,1\rangle$ \\
\hline$x_{5}$ & & & & & $\langle 1,0\rangle$ & $\langle 0.2500,0.9330\rangle$ \\
\hline$x_{6}$ & & & & & & $\langle 1,0\rangle$ \\
\hline
\end{tabular}

Based on the basic principle of MG-PF-DTRSs, a Pythagorean fuzzy decision-making object is approximated over the multi-granulation Pythagorean fuzzy approximation space. So, we give the approach for compute the Pythagorean fuzzy decision-making object from the IMSIS. Then we give the following algorithm for compute the degrees of membership and non-membership of any alternative with respect to a Pythagorean fuzzy decision-making object from the IMSIS:

Algorithm 1 Computation of the degrees of membership and non-membership of any alternative with respect to a Pythagorean fuzzy decision-making object from the IMSIS.

Step 1: First we consider the reasonable membership degree of the domains of the all attributes.

Step 2: If the membership degree of the domain of the attribute $a_{i}=\alpha_{i}$, i.e. $\left(x, a_{i}\right)=\alpha_{i}$, then we write $\mu_{A}\left(x, a_{i}\right)=\alpha_{i}$ and compute $v_{A}\left(x, a_{i}\right)=\sqrt{1-\alpha_{i}^{2}}$. If $\left(x, a_{i}\right)=*$, then we consider $\mu_{A}\left(x, a_{i}\right)=\frac{1}{\left|V_{a_{i}}\right|}$ and $v_{A}\left(x, a_{i}\right)=\sqrt{1-\frac{1}{\left|V_{a_{i}}\right|^{2}}}$.

Step 3: The degrees of membership and non-membership of any $x \in U$ is calculated as follows:

$$
\begin{aligned}
& \mu_{A}(x)=\sum_{i=1}^{t} \frac{\mu_{A(U)}\left(x, a_{i}\right)}{t}, \\
& v_{A}(x)=\sum_{i=1}^{t} \frac{v_{A(U)}\left(x, a_{i}\right)}{t} .
\end{aligned}
$$

For clearance we have the following example:

Example 2 (Continued in Example 1) In this example we find the degrees of membership and non-membership of any alternative with respect to a Pythagorean fuzzy decision-making object from a car IMSIS. According to Algorithm 1, we have

Step 1: Let us we consider the reasonable membership degree of the domains of the all attributes, which is shown in Fig. 2.

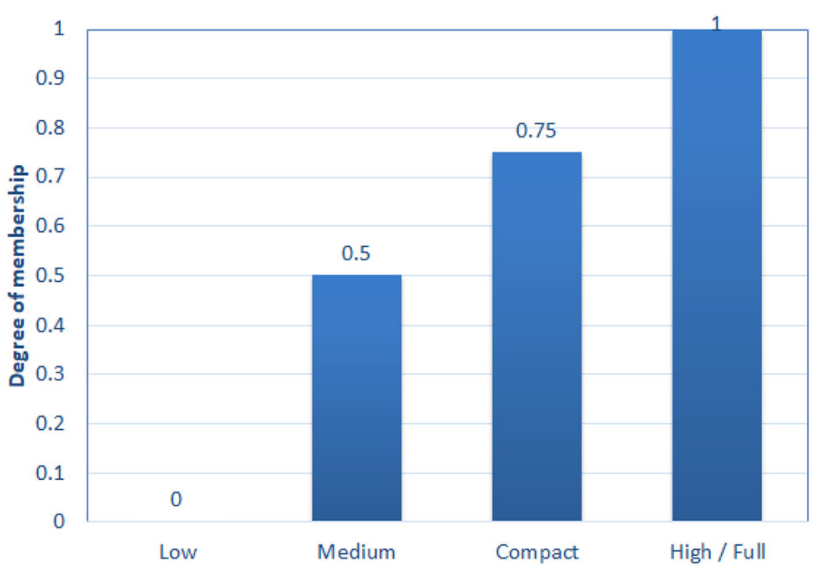

Fig. 2 Membership degree of the domains of the all attributes

Step 2: From Table 3, we get $\left(x_{1}, c_{1}\right)=$ High $=1$, i.e. $\mu_{A}\left(x_{1}, c_{1}\right)=1$, then $v_{A}\left(x_{1}, c_{1}\right)=0$. Similarly, $\mu_{A}\left(x_{1}, c_{2}\right)=0, v_{A}\left(x_{1}, c_{2}\right)=1, \mu_{A}\left(x_{1}, c_{3}\right)=1$, $v_{A}\left(x_{1}, c_{3}\right)=0, \mu_{A}\left(x_{1}, c_{4}\right)=0, v_{A}\left(x_{1}, c_{4}\right)=1$.

Step 3: Using Eqs. (31) and (32), we have $\mu_{A}\left(x_{1}\right)=$ $\frac{1+0+1+0}{4}=0.5$ and $v_{A}\left(x_{1}\right)=\frac{0+1+0+1}{4}=0.5$. Similarly, we also find $\mu_{A}\left(x_{i}\right)$ and $v_{A}\left(x_{i}\right) i=$ $\{2,3, \ldots, 6\}$, which is represented in Eq. (32).

$$
\begin{aligned}
A= & \frac{\langle 0.5,0.5\rangle}{x_{1}}+\frac{\langle 0.25,0.93\rangle}{x_{2}} \\
& +\frac{\langle 0.44,0.89\rangle}{x_{3}}+\frac{\langle 0.88,0.27\rangle}{x_{4}} \\
& +\frac{\langle 0.75,0.43\rangle}{x_{5}}+\frac{\langle 0.65,0.63\rangle}{x_{6}} .
\end{aligned}
$$

\section{An algorithm}

With the help of the results in "MG-PF-DTRSs based on inclusion measure" and "Uncertainty measures", and the discussion in "Decision-making to incomplete multi-source information systems using MG-PF-DTRSs", we design the algorithm of decision-making based on MG-PF-DTRSs and 
their uncertainty measure methods, where the information source is multiple and incomplete. The key steps are elaborated as follows:

Step 1: Suppose that a decision making problem the IMSIS is MSIS $=\left\{\mathrm{IS}_{l} \mid \mathrm{IS}_{l}=\left(U, \mathrm{AT}_{l}, V=\right.\right.$ $\left.\left\{\left(V_{a}\right)_{a \in \mathrm{AT}_{l}}\right\} \cup\{*\}, f_{l}\right\}$. Let us assume that $X_{1}$, $X_{2}, \ldots, X_{r}$ be the subsets of $U$, where the elements of $X_{i}(i=1,2, \ldots, r)$ are randomly selected and not repeat any other elements in $X_{i}(i=1,2, \ldots$, $r)$. To find the best $X_{i}(i=1,2, \ldots, r)$.

Step 2: Computing $R_{k}(1 \leq k \leq l)(U)$ according to Eq. (30).

Step 3: Constructing the Pythagorean fuzzy decisionmaking object $A$ according to Algorithm 1.

Step 4: Choose $\alpha$ and $\beta$.

Step 5: Computing the inclusion measure based TypeI multi-granulation Pythagorean fuzzy $\alpha$-lower approximation

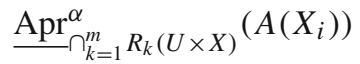

and $\beta$-upper approximation

$\overline{\operatorname{Apr}}_{\cap_{k=1}^{m} R_{k}(U \times X)}^{\beta}\left(A\left(X_{i}\right)\right)$

for each $X_{i} \subseteq U$, respectively.

Step 6: Computing the inclusion measure-based TypeII multi-granulation Pythagorean fuzzy $\alpha$-lower approximation

$\underline{\operatorname{Apr}_{\cup_{k=1}^{\prime}}^{\alpha} R_{k}(U \times X)}\left(A\left(X_{i}\right)\right)$

and $\beta$-upper approximation

$\overline{\mathrm{Apr}}_{\cup_{k=1}^{m} R_{k}(U \times X)}^{\beta}\left(A\left(X_{i}\right)\right)$

for each $X_{i} \subseteq U$, respectively.

Step 7: Computing the inclusion measure-based TypeIII multi-granulation Pythagorean fuzzy $\alpha$-lower approximation

$\bigcap_{k=1}^{m} \underline{\operatorname{Apr}}_{R_{k}(U \times X)}^{\alpha}\left(A\left(X_{i}\right)\right)$

and $\beta$-upper approximation

$\cup_{k=1}^{m} \overline{\operatorname{Apr}}_{R_{k}(U \times X)}^{\beta}\left(A\left(X_{i}\right)\right)$

for each $X_{i} \subseteq U$, respectively.

Step 8: Computing the inclusion measure based TypeIII multi-granulation Pythagorean fuzzy $\alpha$-lower approximation

$\cup_{k=1}^{m} \underline{\operatorname{Apr}}_{R_{k}(U \times X)}^{\alpha}\left(A\left(X_{i}\right)\right)$

and $\beta$-upper approximation

$\cap_{k=1}^{m} \overline{\operatorname{Apr}}_{R_{k}(U \times X)}^{\beta}\left(A\left(X_{i}\right)\right)$

for each $X_{i} \subseteq U$, respectively.

Step 9: Computing $\rho_{\mathrm{I}}, \rho_{\mathrm{II}}, \rho_{\mathrm{III}}$ and $\rho_{\mathrm{IV}}$ using Eqs. (14)(17).

Step 10: Computing $\sigma_{\mathrm{I}}, \sigma_{\mathrm{II}}, \sigma_{\mathrm{III}}$ and $\sigma_{\mathrm{IV}}$ using Eqs. (18)(21).

Step 11: Computing $\omega_{\mathrm{I}}, \omega_{\mathrm{II}}, \omega_{\mathrm{III}}$ and $\omega_{\mathrm{IV}}$ using Eqs. (22)(25).
Step 12: Obtain the best $X_{i}$ according to the higher accuracy, approximation degree and approximation quality for each $X_{i}$.

\section{An illustrative example}

In this subsection, we apply the proposed algorithm to a real decision making. This example is about quick decision making based on a real investment context, under the MGPF-DTRSs and their uncertainty measures models, where the information comes from multiple and incomplete.

\section{Problem description}

The various types of mutual funds (MFs) of different companies listed in the Growth Enterprise Market board of the India Stock Exchange are a popular investment source to an investor as a long-term investment. However, the sufficient knowledge about the various types of MFs of different companies is always not possible for every investor. Our proposed models are effective for those investors. Suppose an investor plans to invest his/her money in MFs of different companies, with the aim of high returns, while he/she has no sufficient knowledge about all MFs, then He/she chooses initially ten MFs according to the past performances, while he/she invests his/her money intpp the best five MFs out of these ten MFs. For making reasonable five MFs out of ten MFs, we have the following decision analysis.

\section{Decision analysis}

We use the algorithm in "An algorithm" of decision analysis based on MG-PF-DTRSs and their uncertainty measure, for decision making.

Suppose an investor initially chooses ten MFs according to the past performances, which is depicted by an IMSIS presented in Table 5. Let $U=\left\{x_{1}, x_{2}, \ldots, x_{10}\right\}$ be a set of ten MFs. Every MF in each sub-information (source) system, denoted by $\mathrm{EC}_{1}, \mathrm{EC}_{2}$, and $\mathrm{EC}_{3}$, is described by attributes. They are $c_{1}=$ Sharpe ratio, $c_{2}=$ Expence ratio, $c_{3}=$ Portfolio concentration ratio, $c_{4}=$ Exit load, $c_{5}=$ Standard deviation, $c_{6}=$ Portfolio turnover ratio, $c_{7}=$ Treynor's ratio, $c_{8}=$ Beta, $c_{9}=$ Fund performance, and $c_{10}=$ Investment philosophy, process and systems followed at the fund house, respectively. The domains of the attributes are: $V_{c_{1}}=\{$ High, Low $\}, V_{c_{2}}=$ Average, Low $\}, V_{c_{3}}=$ \{Moderately low, Moderate $\}, V_{c_{4}}=$ \{Good, Fine $\}, V_{c_{5}}=$ LLow, Moderately low, Moderate, Moderately high, high $\}, V_{c_{6}}=$ \{Good, Fine, Poor $\}, V_{c_{7}}=$ \{Low, Moderately low, Moderate, Moderately high, High\}, $V_{c_{8}}=$ \{Good, Fine, Poor $\}, V_{c_{9}}=$ \{Low, Moderately low, Moderate, Moderately high, High $\}, V_{c_{10}}=\{$ Good, Fine, Poor\}. 
Table 5 Incomplete multi-source information system for ten MFs

\begin{tabular}{|c|c|c|c|c|c|c|c|c|c|c|}
\hline \multirow[t]{2}{*}{$\bar{U}$} & \multicolumn{4}{|l|}{$\underline{\mathrm{EC}_{1}}$} & \multicolumn{3}{|l|}{$\mathrm{EC}_{2}$} & \multicolumn{3}{|l|}{$\mathrm{EC}_{3}$} \\
\hline & $c_{1}$ & $c_{2}$ & $c_{6}$ & $c_{7}$ & $c_{3}$ & $c_{5}$ & $c_{8}$ & $c_{4}$ & $c_{9}$ & $c_{10}$ \\
\hline$x_{1}$ & High & Average & Fine & Moderate & Moderate & Low & Fine & Fine & Moderately high & Fine \\
\hline$x_{2}$ & High & $*$ & Fine & Moderately high & Moderate & $*$ & Fine & Good & High & $*$ \\
\hline$x_{3}$ & Low & $*$ & Fine & $*$ & $*$ & Moderate & $*$ & Good & Moderately low & Good \\
\hline$x_{4}$ & $*$ & Low & Poor & $*$ & $*$ & Moderately low & $*$ & Fine & $*$ & Fine \\
\hline$x_{5}$ & High & Average & Good & Low & $*$ & High & Good & $*$ & High & $*$ \\
\hline$x_{6}$ & $*$ & Low & Fine & $*$ & Moderately low & $*$ & $*$ & Fine & Moderate & $*$ \\
\hline$x_{7}$ & $*$ & $*$ & Good & High & $*$ & $*$ & Fine & $*$ & Moderately high & Fine \\
\hline$x_{8}$ & Low & $*$ & Fine & Moderately high & Moderate & $*$ & Good & Fine & $*$ & $*$ \\
\hline$x_{9}$ & $*$ & Average & Good & $*$ & $*$ & Low & Poor & $*$ & Low & $*$ \\
\hline$x_{10}$ & Low & Low & Fine & $*$ & $*$ & Moderate & $*$ & Fine & Moderate & Fine \\
\hline
\end{tabular}

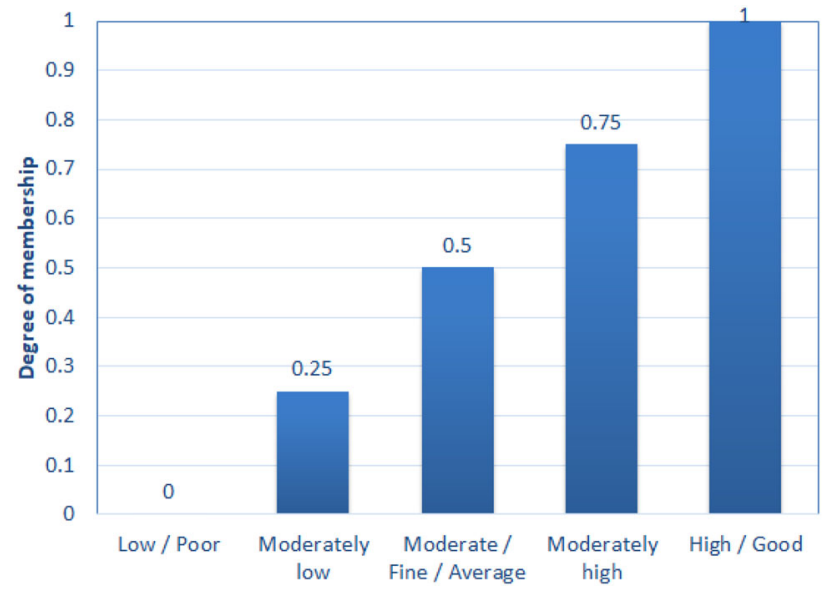

Fig. 3 Membership degree of the domains of the all attributes

For compute $A$ from Table 5, we consider the reasonable membership degree of the domains of the all attributes, which is shown in Fig. 3.

Therefor, we have find $A(U)$ according to Algorithm 1, which is represented in Eq. (34).

$$
\begin{aligned}
A= & \frac{\langle 0.5250,0.7724\rangle}{x_{1}}+\frac{\langle 0.6283,0.6048\rangle}{x_{2}} \\
& +\frac{\langle 0.4783,0.7355\rangle}{x_{3}}+\frac{\langle 0.3483,0.8469\rangle}{x_{4}} \\
& +\frac{\langle 0.6833,0.4541\rangle}{x_{5}}+\frac{\langle 0.3317,0.9287\rangle}{x_{6}} \\
& +\frac{\langle 0.5950,0.6837\rangle}{x_{7}}+\frac{\langle 0.4483,0.8028\rangle}{x_{8}} \\
& +\frac{\langle 0.3533,0.8387\rangle}{x_{9}}+\frac{\langle 0.3533,0.9119\rangle}{x_{10}}
\end{aligned}
$$

Since the investor invest his/her money to the best five MFs out of ten MFs, but there is no other factors which help the investor. He/She has to randomly select five MFs to make investment that means $|X|=5\left(x_{i} \in X\right.$ is a MF between $x_{1}, x_{2}, \ldots, x_{10}$ and not repeat any other MFs in $\left.X\right)$. Suppose $X=\left\{x_{1}, x_{2}, x_{3}, x_{4}, x_{5}\right\}$, in order to facilitate understanding of these model, we exhibit a computational process for each type of MG-PF-DTRS model. Then we calculate the Pythagorean fuzzy decision making object $A(X)$ from $A$ using Eq. (2), which is shown in Eq. (35).

$$
\begin{aligned}
A(X)= & \frac{\langle 0.5250,0.7724\rangle}{x_{1}} \\
& +\frac{\langle 0.6283,0.6048\rangle}{x_{2}}+\frac{\langle 0.4783,0.7355\rangle}{x_{3}} \\
& +\frac{\langle 0.3483,0.8469\rangle}{x_{4}}+\frac{\langle 0.6833,0.4541\rangle}{x_{5}} \\
& +\frac{\langle 0,1\rangle}{x_{6}}+\frac{\langle 0,1\rangle}{x_{7}}+\frac{\langle 0,1\rangle}{x_{8}} \\
& +\frac{\langle 0,1\rangle}{x_{9}}+\frac{\langle 0,1\rangle}{x_{10}} .
\end{aligned}
$$

In the following we have successively obtain for types of MG-PF-DTRSs for $\alpha=0.9$ and $\beta=0.8$.

Type-IMG-PF-DTRSs. First, we compute $\left[x_{i}\right]_{\cap_{k=1}^{3}} R_{k}(U \times U)$ $(i=1,2, \ldots, 10)$ from Tables 6,7 and 8 using the Definition 7. Then we obtain $\left[x_{i}\right]_{\cap_{k=1}^{3} R_{k}(U \times X)}(i=1,2, \ldots, 10)$ using Eq. (6), which is shown in Table 9.

Using Eq. (10), we compute all inclusion measures $I\left(\left[x_{i}\right]_{\cap_{k=1}^{3} R_{k}(U \times X)}, A(X)\right)(1 \leq i \leq 10)$ as follows:

$$
\begin{aligned}
& I\left(\left[x_{1}\right]_{\cap_{k=1}^{3} R_{k}(U \times X)}, A(X)\right)=0.8738, \\
& I\left(\left[x_{2}\right]_{\cap_{k=1}^{3} R_{k}(U \times X)}, A(X)\right)=0.9053, \\
& I\left(\left[x_{3}\right]_{\cap_{k=1}^{3} R_{k}(U \times X)}^{3}, A(X)\right)=0.8749, \\
& I\left(\left[x_{4}\right]_{\cap_{k=1}^{3} R_{k}(U \times X)}, A(X)\right)=0.8499, \\
& I\left(\left[x_{5}\right]_{\cap_{k=1}^{3} R_{k}(U \times X)}, A(X)\right)=0.9265, \\
& I\left(\left[x_{6}\right]_{\cap_{k=1}^{3} R_{k}(U \times X)}, A(X)\right)=1,
\end{aligned}
$$


Table 6 The computing result of $R_{1}(U)$ from $\mathrm{EC}_{1}$

\begin{tabular}{|c|c|c|c|c|c|c|c|c|c|c|}
\hline$R_{1}(U)$ & $x_{1}$ & $x_{2}$ & $x_{3}$ & $x_{4}$ & $x_{5}$ & $x_{6}$ & $x_{7}$ & $x_{8}$ & $x_{9}$ & $x_{10}$ \\
\hline$x_{1}$ & $\begin{array}{l}<1, \\
0>\end{array}$ & $\begin{array}{l}<0.6250 \\
0.4665>\end{array}$ & $\begin{array}{l}<0.4250 \\
0.7115>\end{array}$ & $\begin{array}{l}<0.1750 \\
0.9615>\end{array}$ & $\begin{array}{l}<0.5000 \\
0.5000>\end{array}$ & $\begin{array}{l}<0.4250 \\
0.7115>\end{array}$ & $\begin{array}{l}<0.2500 \\
0.9330>\end{array}$ & $\begin{array}{l}<0.3750 \\
0.7165>\end{array}$ & $\begin{array}{l}<0.4250 \\
0.7115>\end{array}$ & $\begin{array}{l}<0.3000 \\
0.7449>\end{array}$ \\
\hline$x_{2}$ & & $\begin{array}{l}<1 \\
0>\end{array}$ & $\begin{array}{l}<0.3625 \\
0.7370>\end{array}$ & $\begin{array}{l}<0.3000 \\
0.9280>\end{array}$ & $\begin{array}{l}<0.3750 \\
0.7165>\end{array}$ & $\begin{array}{l}<0.5500 \\
0.6780>\end{array}$ & $\begin{array}{l}<0.1875 \\
0.9586>\end{array}$ & $\begin{array}{l}<0.5625 \\
0.4921>\end{array}$ & $\begin{array}{l}<0.3000 \\
0.9280>\end{array}$ & $\begin{array}{l}<0.4250 \\
0.7115>\end{array}$ \\
\hline$x_{3}$ & & & $\begin{array}{l}<1 \\
0>\end{array}$ & $\begin{array}{l}<0.2600 \\
0.9328>\end{array}$ & $\begin{array}{l}<0.1750 \\
0.9615>\end{array}$ & $\begin{array}{l}<0.5100 \\
0.6828>\end{array}$ & $\begin{array}{l}<0.2375 \\
0.9535>\end{array}$ & $\begin{array}{l}<0.6125 \\
0.4870>\end{array}$ & $\begin{array}{l}<0.2600 \\
0.9328>\end{array}$ & $\begin{array}{l}<0.635 \\
0.4663>\end{array}$ \\
\hline$x_{4}$ & & & & $<1$ & $<0.1750$ & $<0.3225$ & $<0.2375$ & $<0.3000$ & $<0.0725$ & $<0.385$ \\
\hline \multirow[t]{2}{*}{$x_{5}$} & & & & $0>$ & $\begin{array}{l}0.9615> \\
<1\end{array}$ & $\begin{array}{l}0.7419> \\
<0.1750\end{array}$ & $\begin{array}{l}0.9535> \\
<0.5000\end{array}$ & $\begin{array}{l}0.9280> \\
<0.1250\end{array}$ & $\begin{array}{l}0.9919> \\
<0.6750\end{array}$ & $\begin{array}{l}0.7163> \\
<0.0500\end{array}$ \\
\hline & & & & & $0>$ & $0.9615>$ & $0.6380>$ & $0.9665>$ & $0.4615>$ & $0.9949>$ \\
\hline \multirow[t]{2}{*}{$x_{6}$} & & & & & & $<1$ & $<0.2375$ & $<0.5500$ & $<0.0725$ & $<0.6350$ \\
\hline & & & & & & $0>$ & $0.9535>$ & $0.6780>$ & $0.9919>$ & $0.4663>$ \\
\hline \multirow[t]{2}{*}{$x_{7}$} & & & & & & & $<1$ & $<0.1875$ & $<0.4875$ & $<0.3000$ \\
\hline & & & & & & & $0>$ & $0.9586>$ & $0.7035>$ & $0.9280>$ \\
\hline \multirow[t]{2}{*}{$x_{8}$} & & & & & & & & $<1$ & $<0.3000$ & $<0.6750$ \\
\hline & & & & & & & & $0>$ & $0.9280>$ & $0.4615>$ \\
\hline \multirow[t]{2}{*}{$x_{9}$} & & & & & & & & & $<1$, & $<0.1350$ \\
\hline & & & & & & & & & $0>$ & $0.9663>$ \\
\hline \multirow[t]{2}{*}{$x_{10}$} & & & & & & & & & & $<1$ \\
\hline & & & & & & & & & & $0>$ \\
\hline
\end{tabular}

Table 7 The computing result of $R_{2}(U)$ from $\mathrm{EC}_{2}$

\begin{tabular}{|c|c|c|c|c|c|c|c|c|c|c|}
\hline$R_{2}(U)$ & $x_{1}$ & $x_{2}$ & $x_{3}$ & $x_{4}$ & $x_{5}$ & $x_{6}$ & $x_{7}$ & $x_{8}$ & $x_{9}$ & $x_{10}$ \\
\hline$x_{1}$ & $\begin{array}{l}<1 \\
0>\end{array}$ & $\begin{array}{l}<0.7333 \\
0.3266>\end{array}$ & $\begin{array}{l}<0.2778 \\
0.9363>\end{array}$ & $\begin{array}{l}<0.2778 \\
0.9363>\end{array}$ & $\begin{array}{l}<0.1667 \\
0.9553>\end{array}$ & $\begin{array}{l}<0.1778 \\
0.9742>\end{array}$ & $\begin{array}{l}<0.5667 \\
0.6153>\end{array}$ & $\begin{array}{l}<0.4000 \\
0.6599>\end{array}$ & $\begin{array}{l}<0.5000 \\
0.6220>\end{array}$ & $\begin{array}{l}<0.2778 \\
0.9363>\end{array}$ \\
\hline$x_{2}$ & & $\begin{array}{l}<1 \\
0>\end{array}$ & $\begin{array}{l}<0.3444 \\
0.9295>\end{array}$ & $\begin{array}{l}<0.3444 \\
0.9295>\end{array}$ & $\begin{array}{l}<0.2333 \\
0.9486>\end{array}$ & $\begin{array}{l}<0.1244 \\
0.9807>\end{array}$ & $\begin{array}{l}<0.5133 \\
0.6217>\end{array}$ & $\begin{array}{l}<0.3467 \\
0.6664>\end{array}$ & $\begin{array}{l}<0.2333 \\
0.9486>\end{array}$ & $\begin{array}{l}<0.3444, \\
0.9295>\end{array}$ \\
\hline$x_{3}$ & & & $\begin{array}{l}<1 \\
0>\end{array}$ & $\begin{array}{l}<0.1204 \\
0.9874>\end{array}$ & $\begin{array}{l}<0.1944 \\
0.9704>\end{array}$ & $\begin{array}{l}<0.2704 \\
0.9465>\end{array}$ & $\begin{array}{l}<0.2611 \\
0.9636>\end{array}$ & $\begin{array}{l}<0.3444 \\
0.9295>\end{array}$ & $\begin{array}{l}<0.1944 \\
0.9704>\end{array}$ & $\begin{array}{l}<0.4537 \\
0.6540>\end{array}$ \\
\hline$x_{4}$ & & & & $\begin{array}{l}<1 \\
0>\end{array}$ & $\begin{array}{l}<0.1204 \\
0.9874>\end{array}$ & $\begin{array}{l}<0.2704 \\
0.9465>\end{array}$ & $\begin{array}{l}<0.2611 \\
0.9636>\end{array}$ & $\begin{array}{l}<0.3444 \\
0.9295>\end{array}$ & $\begin{array}{l}<0.1944 \\
0.9704>\end{array}$ & $\begin{array}{l}<0.1204, \\
0.9874>\end{array}$ \\
\hline$x_{5}$ & & & & & $\begin{array}{l}<1 \\
0>\end{array}$ & $\begin{array}{l}<0.3444 \\
0.9295>\end{array}$ & $\begin{array}{l}<0.2611 \\
0.9636>\end{array}$ & $\begin{array}{l}<0.5667 \\
0.6153>\end{array}$ & $\begin{array}{l}<0.0833 \\
0.9894>\end{array}$ & $\begin{array}{l}<0.1944, \\
0.9704>\end{array}$ \\
\hline$x_{6}$ & & & & & & $\begin{array}{l}<1 \\
0>\end{array}$ & $\begin{array}{l}<0.2911 \\
0.9360>\end{array}$ & $\begin{array}{l}<0.1244 \\
0.9807>\end{array}$ & $\begin{array}{l}<0.3444 \\
0.9295>\end{array}$ & $\begin{array}{l}<0.2704 \\
0.9465>\end{array}$ \\
\hline$x_{7}$ & & & & & & & $\begin{array}{l}<1 \\
0>\end{array}$ & $\begin{array}{l}<0.1800 \\
0.9551>\end{array}$ & $\begin{array}{l}<0.1500 \\
0.9827>\end{array}$ & $\begin{array}{l}<0.2611, \\
0.9636>\end{array}$ \\
\hline$x_{8}$ & & & & & & & & $\begin{array}{l}<1 \\
0>\end{array}$ & $\begin{array}{l}<0.2333 \\
0.9486>\end{array}$ & $\begin{array}{l}<0.2333, \\
0.9486>\end{array}$ \\
\hline$x_{9}$ & & & & & & & & & $\begin{array}{l}<1 \\
0>\end{array}$ & $\begin{array}{l}<0.1944 \\
0.9704>\end{array}$ \\
\hline$x_{10}$ & & & & & & & & & & $\begin{array}{l}<1 \\
0>\end{array}$ \\
\hline
\end{tabular}


Table 8 The computing result of $R_{3}(U)$ from $\mathrm{EC}_{3}$

\begin{tabular}{|c|c|c|c|c|c|c|c|c|c|c|}
\hline$R_{3}(U)$ & $x_{1}$ & $x_{2}$ & $x_{3}$ & $x_{4}$ & $x_{5}$ & $x_{6}$ & $x_{7}$ & $x_{8}$ & $x_{9}$ & $x_{10}$ \\
\hline$x_{1}$ & $\begin{array}{l}<1, \\
0>\end{array}$ & $\begin{array}{l}<0.1111 \\
0.9809>\end{array}$ & $\begin{array}{l}<0 \\
1>\end{array}$ & $\begin{array}{l}<0.7333 \\
0.3266>\end{array}$ & $\begin{array}{l}<0.2778 \\
0.9363>\end{array}$ & $\begin{array}{l}<0.4444 \\
0.6476>\end{array}$ & $\begin{array}{l}<0.8333 \\
0.2887>\end{array}$ & $\begin{array}{l}<0.5111 \\
0.6409>\end{array}$ & $\begin{array}{l}<0.2778 \\
0.9363>\end{array}$ & $\begin{array}{l}<0.6667, \\
0.3333>\end{array}$ \\
\hline$x_{2}$ & & $\begin{array}{l}<1 \\
0>\end{array}$ & $\begin{array}{l}<0.4444 \\
0.6476>\end{array}$ & $\begin{array}{l}<0.1778 \\
0.9742>\end{array}$ & $\begin{array}{l}<0.5370 \\
0.6199>\end{array}$ & $\begin{array}{l}<0.3704 \\
0.6646>\end{array}$ & $\begin{array}{l}<0.2778 \\
0.9363>\end{array}$ & $\begin{array}{l}<0.1037 \\
0.9912>\end{array}$ & $\begin{array}{l}<0.2704 \\
0.9465>\end{array}$ & $\begin{array}{l}<0.1111 \\
0.9809>\end{array}$ \\
\hline$x_{3}$ & & & $\begin{array}{l}<1 \\
0>\end{array}$ & $\begin{array}{l}<0.0667 \\
0.9933>\end{array}$ & $\begin{array}{l}<0.2778 \\
0.9363>\end{array}$ & $\begin{array}{l}<0.1111 \\
0.9809>\end{array}$ & $\begin{array}{l}<0.1667 \\
0.9553>\end{array}$ & $\begin{array}{l}<0.1778 \\
0.9742>\end{array}$ & $\begin{array}{l}<0.2778 \\
0.9363>\end{array}$ & $\begin{array}{l}<0 \\
1>\end{array}$ \\
\hline$x_{4}$ & & & & $\begin{array}{l}<1 \\
0>\end{array}$ & $\begin{array}{l}<0.3444 \\
0.9295>\end{array}$ & $\begin{array}{l}<0.5111 \\
0.6409>\end{array}$ & $\begin{array}{l}<0.5667 \\
0.6153>\end{array}$ & $\begin{array}{l}<0.4578 \\
0.6473>\end{array}$ & $\begin{array}{l}<0.3444 \\
0.9295>\end{array}$ & $\begin{array}{l}<0.7333 \\
0.3266>\end{array}$ \\
\hline$x_{5}$ & & & & & $\begin{array}{l}<1 \\
0>\end{array}$ & $\begin{array}{l}<0.2037 \\
0.9533>\end{array}$ & $\begin{array}{l}<0.1944 \\
0.9704>\end{array}$ & $\begin{array}{l}<0.2704 \\
0.9465>\end{array}$ & $\begin{array}{l}<0.1204 \\
0.9874>\end{array}$ & $\begin{array}{l}<0.1944, \\
0.9704>\end{array}$ \\
\hline$x_{6}$ & & & & & & $\begin{array}{l}<1 \\
0>\end{array}$ & $\begin{array}{l}<0.2778 \\
0.9363>\end{array}$ & $\begin{array}{l}<0.4370 \\
0.6579>\end{array}$ & $\begin{array}{l}<0.2037 \\
0.9533>\end{array}$ & $\begin{array}{l}<0.7778 \\
0.3143>\end{array}$ \\
\hline$x_{7}$ & & & & & & & $\begin{array}{l}<1 \\
0>\end{array}$ & $\begin{array}{l}<0.3444 \\
0.9295>\end{array}$ & $\begin{array}{l}<0.1944 \\
0.9704>\end{array}$ & $\begin{array}{l}<0.5000 \\
0.6220>\end{array}$ \\
\hline$x_{8}$ & & & & & & & & $\begin{array}{l}<1 \\
0>\end{array}$ & $\begin{array}{l}<0.2704 \\
0.9465>\end{array}$ & $\begin{array}{l}<0.5111, \\
0.6409>\end{array}$ \\
\hline$x_{9}$ & & & & & & & & & $\begin{array}{l}<1 \\
0>\end{array}$ & $\begin{array}{l}<0.2778 \\
0.9363>\end{array}$ \\
\hline$x_{10}$ & & & & & & & & & & $\begin{array}{l}<1 \\
0>\end{array}$ \\
\hline
\end{tabular}

Table 9 The computing result of $\left[x_{i}\right]_{\cap_{k=1}^{3} R_{k}(U \times X)}$

\begin{tabular}{|c|c|c|c|c|c|c|c|c|c|c|}
\hline$\left[x_{i}\right]_{\cap_{k=1}^{3}} R_{k}(U \times X)$ & $x_{1}$ & $x_{2}$ & $x_{3}$ & $x_{4}$ & $x_{5}$ & $x_{6}$ & $x_{7}$ & $x_{8}$ & $x_{9}$ & $x_{10}$ \\
\hline$x_{1}$ & $\begin{array}{l}<1 \\
0>\end{array}$ & $\begin{array}{l}<0.1111 \\
0.9809>\end{array}$ & $\begin{array}{l}<0 \\
1>\end{array}$ & $\begin{array}{l}<0.1750 \\
0.9615>\end{array}$ & $\begin{array}{l}<0.1667 \\
0.9553>\end{array}$ & $\begin{array}{l}<0 \\
1>\end{array}$ & $\begin{array}{l}<0 \\
1>\end{array}$ & $\begin{array}{l}<0, \\
1>\end{array}$ & $\begin{array}{l}<0 \\
1>\end{array}$ & $\begin{array}{l}<0, \\
1>\end{array}$ \\
\hline$x_{2}$ & $\begin{array}{l}<0.1111 \\
0.9809>\end{array}$ & $\begin{array}{l}<1 \\
0>\end{array}$ & $\begin{array}{l}<0.3444 \\
0.9295>\end{array}$ & $\begin{array}{l}<0.1778 \\
0.9742>\end{array}$ & $\begin{array}{l}<0.2333 \\
0.9486>\end{array}$ & $\begin{array}{l}<0 \\
1>\end{array}$ & $\begin{array}{l}<0 \\
1>\end{array}$ & $\begin{array}{l}<0, \\
1>\end{array}$ & $\begin{array}{l}<0 \\
1>\end{array}$ & $\begin{array}{l}<0, \\
1>\end{array}$ \\
\hline$x_{3}$ & $\begin{array}{l}<0 \\
1>\end{array}$ & $\begin{array}{l}<0.3444 \\
0.9295>\end{array}$ & $\begin{array}{l}<1 \\
0>\end{array}$ & $\begin{array}{l}<0.0667 \\
0.9933>\end{array}$ & $\begin{array}{l}<0.1750 \\
0.9704>\end{array}$ & $\begin{array}{l}<0 \\
1>\end{array}$ & $\begin{array}{l}<0 \\
1>\end{array}$ & $\begin{array}{l}<0, \\
1>\end{array}$ & $\begin{array}{l}<0 \\
1>\end{array}$ & $\begin{array}{l}<0, \\
1>\end{array}$ \\
\hline$x_{4}$ & $\begin{array}{l}<0.1750 \\
0.9615>\end{array}$ & $\begin{array}{l}<0.1778 \\
0.9742>\end{array}$ & $\begin{array}{l}<0.0667 \\
0.9933>\end{array}$ & $\begin{array}{l}<1 \\
0>\end{array}$ & $\begin{array}{l}<0.1204 \\
0.9874>\end{array}$ & $\begin{array}{l}<0 \\
1>\end{array}$ & $\begin{array}{l}<0 \\
1>\end{array}$ & $\begin{array}{l}<0, \\
1>\end{array}$ & $\begin{array}{l}<0 \\
1>\end{array}$ & $\begin{array}{l}<0, \\
1>\end{array}$ \\
\hline$x_{5}$ & $\begin{array}{l}<0.1667 \\
0.9553>\end{array}$ & $\begin{array}{l}<0.2333 \\
0.9486>\end{array}$ & $\begin{array}{l}<0.1750 \\
0.9704>\end{array}$ & $\begin{array}{l}<0.1204 \\
0.9874>\end{array}$ & $\begin{array}{l}<1 \\
0>\end{array}$ & $\begin{array}{l}<0 \\
1>\end{array}$ & $\begin{array}{l}<0 \\
1>\end{array}$ & $\begin{array}{l}<0, \\
1>\end{array}$ & $\begin{array}{l}<0 \\
1>\end{array}$ & $\begin{array}{l}<0, \\
1>\end{array}$ \\
\hline$x_{6}$ & $\begin{array}{l}<0.1778 \\
0.9742>\end{array}$ & $\begin{array}{l}<0.1244 \\
0.9807>\end{array}$ & $\begin{array}{l}<0.1111 \\
0.9809>\end{array}$ & $\begin{array}{l}<0.2704 \\
0.9465>\end{array}$ & $\begin{array}{l}<0.1750 \\
0.9615>\end{array}$ & $\begin{array}{l}<0 \\
1>\end{array}$ & $\begin{array}{l}<0 \\
1>\end{array}$ & $\begin{array}{l}<0, \\
1>\end{array}$ & $\begin{array}{l}<0 \\
1>\end{array}$ & $\begin{array}{l}<0, \\
1>\end{array}$ \\
\hline$x_{7}$ & $\begin{array}{l}<0.2500 \\
0.9330>\end{array}$ & $\begin{array}{l}<0.1875 \\
0.9586>\end{array}$ & $\begin{array}{l}<0.1667 \\
0.9636>\end{array}$ & $\begin{array}{l}<0.2375 \\
0.9636>\end{array}$ & $\begin{array}{l}<0.1944 \\
0.9704>\end{array}$ & $\begin{array}{l}<0 \\
1>\end{array}$ & $\begin{array}{l}<0 \\
1>\end{array}$ & $\begin{array}{l}<0, \\
1>\end{array}$ & $\begin{array}{l}<0 \\
1>\end{array}$ & $\begin{array}{l}<0, \\
1>\end{array}$ \\
\hline$x_{8}$ & $\begin{array}{l}<0.3750 \\
0.7165>\end{array}$ & $\begin{array}{l}<0.1037 \\
0.9912>\end{array}$ & $\begin{array}{l}<0.1778 \\
0.9742>\end{array}$ & $\begin{array}{l}<0.3000 \\
0.9295>\end{array}$ & $\begin{array}{l}<0.1250 \\
0.9665>\end{array}$ & $\begin{array}{l}<0 \\
1>\end{array}$ & $\begin{array}{l}<0 \\
1>\end{array}$ & $\begin{array}{l}<0, \\
1>\end{array}$ & $\begin{array}{l}<0 \\
1>\end{array}$ & $\begin{array}{l}<0, \\
1>\end{array}$ \\
\hline$x_{9}$ & $\begin{array}{l}<0.2778 \\
0.9363>\end{array}$ & $\begin{array}{l}<0.2333 \\
0.9486>\end{array}$ & $\begin{array}{l}<0.1944 \\
0.9704>\end{array}$ & $\begin{array}{l}<0.0725 \\
0.9919>\end{array}$ & $\begin{array}{l}<0.0833 \\
0.9894>\end{array}$ & $\begin{array}{l}<0 \\
0>\end{array}$ & $\begin{array}{l}<0 \\
1>\end{array}$ & $\begin{array}{l}<0, \\
1\end{array}$ & $\begin{array}{l}<0 \\
1>\end{array}$ & $\begin{array}{l}<0, \\
1>\end{array}$ \\
\hline$x_{10}$ & $\begin{array}{l}<0.2778 \\
0.9363>\end{array}$ & $\begin{array}{l}<0.1111 \\
0.9809>\end{array}$ & $\begin{array}{l}<0 \\
1>\end{array}$ & $\begin{array}{l}<0.1204 \\
0.9874>\end{array}$ & $\begin{array}{l}<0.0500 \\
0.9949>\end{array}$ & $\begin{array}{l}<0 \\
1>\end{array}$ & $\begin{array}{l}<0 \\
1>\end{array}$ & $\begin{array}{l}<0, \\
1>\end{array}$ & $\begin{array}{l}<0 \\
1>\end{array}$ & $\begin{array}{l}<0, \\
1>\end{array}$ \\
\hline
\end{tabular}


$I\left(\left[x_{7}\right]_{\cap_{k=1}^{3} R_{k}(U \times X)}, A(X)\right)=1$,

$I\left(\left[x_{8}\right]_{\cap_{k=1}^{3} R_{k}(U \times X)}, A(X)\right)=0.9913$,

$I\left(\left[x_{9}\right]_{\cap_{k=1}^{3} R_{k}(U \times X)}, A(X)\right)=1$,

$I\left(\left[x_{10}\right]_{\cap_{k=1}^{3} R_{k}(U \times X)}, A(X)\right)=1$.

For $\alpha=0.9$ and $\beta=0.8$ we obtain

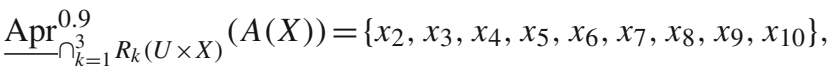

$\overline{\operatorname{Apr}}_{\cap_{k=1}^{m} R_{k}(U \times X)}^{0.8}(A(X))=U$.

and

$\operatorname{POS}^{0.9}(A(X))=\left\{x_{2}, x_{3}, x_{4}, x_{5}, x_{6}, x_{7}, x_{8}, x_{9}, x_{10}\right\}$,

$\mathrm{NEG}^{0.8}(A(X))=\emptyset$,

$\operatorname{BND}^{(0.9,0.8)}(A(X))=\left\{x_{1}\right\}$.

Type-IIMG-PF-DTRSs First, we compute $\left[x_{i}\right]_{\cup_{k=1}^{3}} R_{k}(U \times U)$ ( $i=1,2, \ldots, 10)$ from Tables 6,7 and 8 using the Definition 7. Then we obtain $\left[x_{i}\right]_{\cup_{k=1}^{3} R_{k}(U \times X)}(i=1,2, \ldots, 10)$ using Eq. (6), which is not display here. Using Eq. (10), we compute all inclusion measures $I\left(\left[x_{i}\right]_{\cup_{k=1}^{3} R_{k}(U \times X)}, A(X)\right)$ $(1 \leq i \leq 10)$ as follows:

$I\left(\left[x_{1}\right]_{\cup_{k=1}^{3} R_{k}(U \times X)}, A(X)\right)=0.7209$,
$I\left(\left[x_{2}\right]_{\cup_{k=1}^{3} R_{k}(U \times X)}, A(X)\right)=0.8135$,
$I\left(\left[x_{3}\right]_{\cup_{k=1}^{3} R_{k}(U \times X)}, A(X)\right)=0.8573$,
$I\left(\left[x_{4}\right]_{\cup_{k=1}^{3} R_{k}(U \times X)}, A(X)\right)=0.7816$,
$I\left(\left[x_{5}\right]_{\cup_{k=1}^{3} R_{k}(U \times X)}, A(X)\right)=0.8876$,
$I\left(\left[x_{6}\right]_{\cup_{k=1}^{3} R_{k}(U \times X)}, A(X)\right)=0.9215$,
$I\left(\left[x_{7}\right]_{\cup_{k=1}^{3} R_{k}(U \times X)}, A(X)\right)=0.8470$,
$I\left(\left[x_{8}\right]_{\cup_{k=1}^{3} R_{k}(U \times X)}, A(X)\right)=0.8738$,
$I\left(\left[x_{9}\right]_{\cup_{k=1}^{3} R_{k}(U \times X)}, A(X)\right)=0.9777$,
$I\left(\left[x_{10}\right]_{\cup_{k=1}^{3} R_{k}(U \times X)}, A(X)\right)=0.7800$.

For $\alpha=0.9$ and $\beta=0.8$ we obtain

$\mathrm{Apr}_{\cup_{k=1}^{3} R_{k}(U \times X)}^{0.9}(A(X))=\left\{x_{6}, x_{9}\right\}$,

$\overline{\mathrm{Apr}}_{\cup_{k=1}^{m} R_{k}(U \times X)}^{0.8}(A(X))=\left\{x_{2}, x_{3}, x_{5}, x_{6}, x_{7}, x_{8}, x_{9}, x_{10}\right\}$.

and

$\operatorname{POS}^{0.9}(A(X))=\left\{x_{6}, x_{9}\right\}$,

$\mathrm{NEG}^{0.8}(A(X))=\left\{x_{1}, x_{4}\right\}$,

$\operatorname{BND}^{(0.9,0.8)}(A(X))=\left\{x_{2}, x_{3}, x_{5}, x_{7}, x_{8}\right\}$.
Type-III MG-PF-DTRSs First we compute $R_{1}(U \times X)$, $R_{2}(U \times X)$ and $R_{3}(U \times X)$ from Tables 6,7 and 8 , and $I\left(\left[x_{i}\right]_{R_{1}(U \times X)}, A(X)\right), I\left(\left[x_{i}\right]_{R_{2}(U \times X)}, A(X)\right)$ and $I\left(\left[x_{i}\right]_{R_{3}(U \times X)}, A(X)\right)$ for $(1 \leq i \leq 10)$, which are not displayed here. Using Eqs. (12) and (13), we compute all inclusion measures $\cap_{k=1}^{3} I\left(\left[x_{i}\right]_{R_{k}(U \times X)}, A(X)\right)$ and $\cup_{k=1}^{3} I\left(\left[x_{i}\right]_{R_{k}(U \times X)}, A(X)\right)$ for $(1 \leq i \leq 10)$ as follows:

$\cap_{k=1}^{3} I\left(\left[x_{1}\right]_{R_{k}(U \times X)}, A(X)\right)=0.7822$,

$\cap_{k=1}^{3} I\left(\left[x_{2}\right]_{R_{k}(U \times X)}, A(X)\right)=0.8346$,

$\cap_{k=1}^{3} I\left(\left[x_{3}\right]_{R_{k}(U \times X)}, A(X)\right)=0.8581$,

$\cap_{k=1}^{3} I\left(\left[x_{4}\right]_{R_{k}(U \times X)}, A(X)\right)=0.7827$,

$\cap_{k=1}^{3} I\left(\left[x_{5}\right]_{R_{k}(U \times X)}, A(X)\right)=0.8871$,

$\cap_{k=1}^{3} I\left(\left[x_{6}\right]_{R_{k}(U \times X)}, A(X)\right)=0.9328$,

$\cap_{k=1}^{3} I\left(\left[x_{7}\right]_{R_{k}(U \times X)}, A(X)\right)=0.8559$,

$\cap_{k=1}^{3} I\left(\left[x_{8}\right]_{R_{k}(U \times X)}, A(X)\right)=0.9289$,

$\cap_{k=1}^{3} I\left(\left[x_{9}\right]_{R_{k}(U \times X)}, A(X)\right)=0.9785$,

$\cap_{k=1}^{3} I\left(\left[x_{10}\right]_{R_{k}(U \times X)}, A(X)\right)=0.8360$,

and $\cup_{k=1}^{3} I\left(\left[x_{1}\right]_{R_{k}(U \times X)}, A(X)\right)=0.8422$,

$\cup_{k=1}^{3} I\left(\left[x_{2}\right]_{R_{k}(U \times X)}, A(X)\right)=0.8877$,

$\cup_{k=1}^{3} I\left(\left[x_{3}\right]_{R_{k}(U \times X)}, A(X)\right)=0.8744$,

$\cup_{k=1}^{3} I\left(\left[x_{4}\right]_{R_{k}(U \times X)}, A(X)\right)=0.8499$,

$\cup_{k=1}^{3} I\left(\left[x_{5}\right]_{R_{k}(U \times X)}, A(X)\right)=0.9266$,

$\cup_{k=1}^{3} I\left(\left[x_{6}\right]_{R_{k}(U \times X)}, A(X)\right)=1$,

$\cup_{k=1}^{3} I\left(\left[x_{7}\right]_{R_{k}(U \times X)}, A(X)\right)=1$,

$\cup_{k=1}^{3} I\left(\left[x_{8}\right]_{R_{k}(U \times X)}, A(X)\right)=0.9281$,

$\cup_{k=1}^{3} I\left(\left[x_{9}\right]_{R_{k}(U \times X)}, A(X)\right)=1$,

$\cup_{k=1}^{3} I\left(\left[x_{10}\right]_{R_{k}(U \times X)}, A(X)\right)=0.9883$.

For $\alpha=0.9$ and $\beta=0.8$ we obtain

$\cap_{k=1}^{3}{\stackrel{\operatorname{Apr}}{0.9} R_{k}(U \times X)}_{(A(X))=\left\{x_{6}, x_{8}, 9\right\}}$,

$\cup_{k=1}^{3} \overline{\operatorname{Apr}}_{R_{k}(U \times X)}^{0.8}(A(X))=U$.

and

$\operatorname{POS}^{0.9}(A(X))=\left\{x_{6}, x_{8}, x_{9}\right\}$,

$\mathrm{NEG}^{0.8}(A(X))=\emptyset$,

$\operatorname{BND}^{(0.9,0.8)}(A(X))=\left\{x_{1}, x_{2}, x_{3}, x_{4}, x_{5}, x_{7}, x_{10}\right\}$.

Type-IV MG-PF-DTRSs From inclusion measures $\cap_{k=1}^{3} I\left(\left[x_{i}\right]_{R_{k}(U \times X)}, A(X)\right)$ and $\cup_{k=1}^{3} I\left(\left[x_{i}\right]_{R_{k}(U \times X)}, A(X)\right)$ for $(1 \leq i \leq 10)$ we have obtained Type-IV MG-PF-DTRSs for $\alpha=0.9$ and $\beta=0.8$ as follows:

$\cup_{k=1}^{3} \underline{\operatorname{Apr}}_{R_{k}(U \times X)}^{0.9}(A(X))=\left\{x_{5}, x_{6}, x_{7}, x_{8}, x_{9}, x_{10}\right\}$,

$\cap_{k=1}^{3} \overline{\operatorname{Apr}}_{R_{k}(U \times X)}^{0.8}(A(X))=\left\{x_{2}, x_{3}, x_{4}, x_{5}, x_{6}, x_{7}, x_{8}, x_{9}, x_{10}\right\}$. 
Table 10 The results of uncertainty measures

\begin{tabular}{|c|c|c|c|c|c|c|c|c|c|c|c|c|c|}
\hline \multirow[t]{2}{*}{ No. } & \multirow[t]{2}{*}{$X$} & \multicolumn{3}{|c|}{ Type-I MG-PF-DTRSs } & \multicolumn{3}{|c|}{ Type-II MG-PF-DTRSs } & \multicolumn{3}{|c|}{ Type-III MG-PF-DTRSs } & \multicolumn{3}{|c|}{ Type-IV MG-PF-DTRSs } \\
\hline & & $\rho_{\mathrm{I}}$ & $\sigma_{\mathrm{I}}$ & $\omega_{\mathrm{I}}$ & $\rho_{\mathrm{II}}$ & $\sigma_{\mathrm{II}}$ & $\omega_{\mathrm{II}}$ & $\rho_{\mathrm{III}}$ & $\sigma_{\mathrm{III}}$ & $\omega_{\text {III }}$ & $\rho_{\mathrm{IV}}$ & $\sigma_{\mathrm{IV}}$ & $\omega_{\mathrm{IV}}$ \\
\hline 1 & $x_{1,2,3,4,5}$ & 0.7 & 1.4 & 0.7 & 0.2857 & 0.4 & 0.2 & 0.3 & 0.6 & 0.3 & 0.75 & 1.2 & 0.6 \\
\hline 2 & $x_{1,2,5,6,7}$ & 0.7 & 1.4 & 0.7 & 0.5 & 0.6 & 0.3 & 0.3 & 0.6 & 0.3 & 0.75 & 1.2 & 0.6 \\
\hline 3 & $x_{1,3,5,7,8}$ & 0.6 & 1.2 & 0.6 & 0.25 & 0.4 & 0.2 & 0.2 & 0.4 & 0.2 & 0.6667 & 1.2 & 0.6 \\
\hline 4 & $x_{1,3,4,6,7}$ & 0.5 & 1 & 0.5 & 0.5 & 0.4 & 0.2 & 0.2 & 0.4 & 0.2 & 1 & 1 & 0.5 \\
\hline 5 & $x_{2,4,5,6,7}$ & 0.7 & 1.4 & 0.7 & 0.5 & 0.6 & 0.3 & 0.4 & 0.8 & 0.4 & 1 & 1.2 & 0.6 \\
\hline 6 & $x_{2,4,5,9,10}$ & 0.6 & 1.2 & 0.6 & 0.1429 & 0.2 & 0.1 & 0.1 & 0.2 & 0.1 & 0.875 & 1.4 & 0.6 \\
\hline 7 & $x_{2,7,8,9,10}$ & 0.6 & 1.2 & 0.6 & 0 & 0 & 0 & 0.1 & 0.2 & 0.1 & 0.8571 & 1.2 & 0.6 \\
\hline 8 & $x_{3,4,6,8,9}$ & 0.5 & 1 & 0.5 & 0.25 & 0.2 & 0.1 & 0.2 & 0.4 & 0.2 & 1 & 1 & 0.5 \\
\hline 9 & $x_{3,5,6,9,10}$ & 0.6 & 1.2 & 0.6 & 0.2 & 0.2 & 0.1 & 0.1 & 0.2 & 0.1 & 0.8571 & 1.2 & 0.6 \\
\hline 10 & $x_{4,6,7,8,9}$ & 0.5 & 1 & 0.5 & 0 & 0 & 0 & 0.1 & 0.2 & 0.1 & 1 & 1 & 0.5 \\
\hline
\end{tabular}

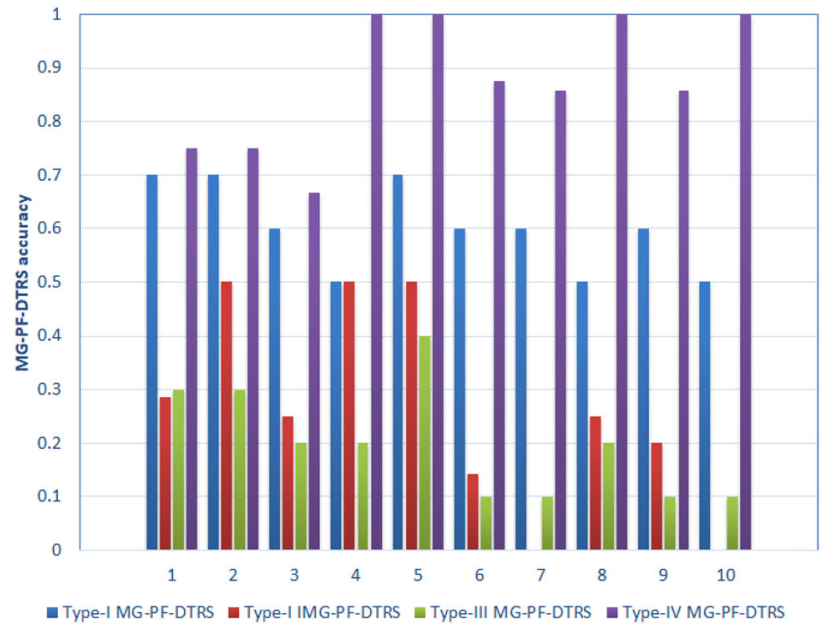

Fig. 4 A comparison of the accuracy

and

$\operatorname{POS}^{0.9}(A(X))=\left\{x_{5}, x_{6}, x_{7}, x_{8}, x_{9}, x_{10}\right\}$,

$\mathrm{NEG}^{0.8}(A(X))=\left\{x_{1}\right\}$,

$\operatorname{BND}^{(0.9,0.8)}(A(X))=\left\{x_{2}, x_{3}\right\}$.

It is obvious that these four types of results are not entirely consistent. Then, the uncertainties are not entirely consistent for them and several kinds of uncertainty measure methods are necessary. Consequently, in different fields should select different model according the different requirements in practical application. Based on the calculated approximation sets, we can measure the uncertainty of the alternative MFs to estimate the investment. To evaluate the performance of the proposed uncertainty measure methods, we conduct a series of experiments to calculate these three uncertainty measures. Therefor, in our experiment, we randomly select five MFs from the set $U$, and uncertainty evolution's for them as shown in Table 10.

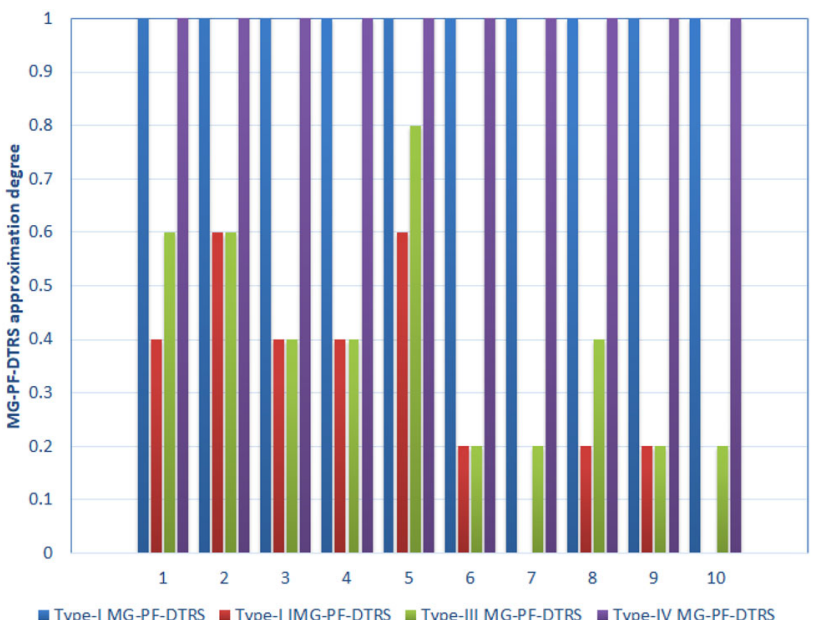

Fig. 5 A comparison of the approximation degree

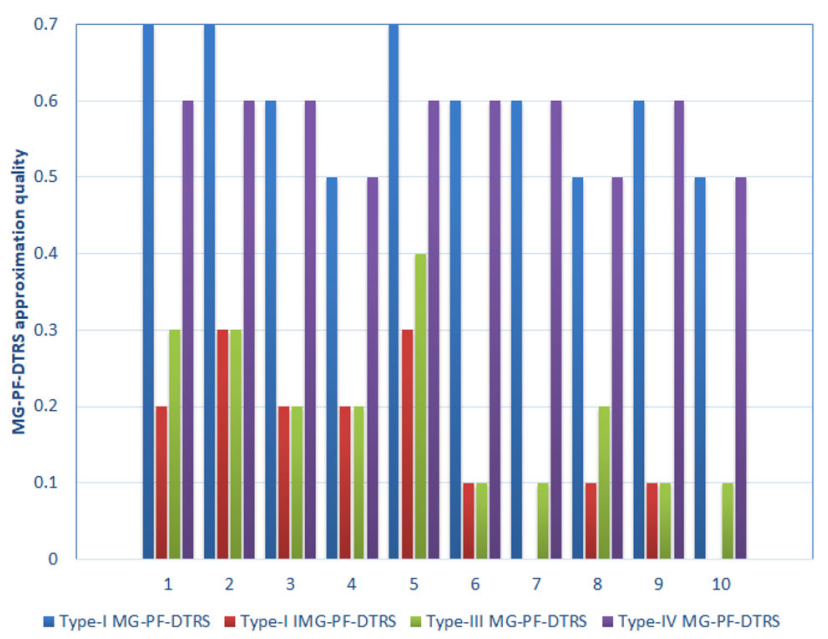

Fig. 6 A comparison of the approximation quality

According to Table 10 and Figs. 4, 5 and 6 we can get that the fifth set $X=\left\{x_{2}, x_{4}, x_{5}, x_{6}, x_{7}\right\}$ with higher accuracy, approximation degree and approximation quality for each

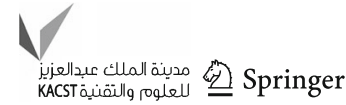


model. That is, $x_{2}, x_{4}, x_{5}, x_{6}$ and $x_{7}$ are the best five MFs out of ten MFs for the investors to invest. There is no doubt that there are more recommended programs, but its best in the given ten options.

\section{Conclusions}

In this paper, we present four types of MG-PF-DTRSs of Pythagorean fuzzy subset (of a subset of the given universe) of the PFS (of the given universe) and study their uncertainty measure methods based on the Pythagorean fuzzy inclusion measure within the framework of multi-granulation Pythagorean fuzzy approximation space. Using this four types of MG-PF-DTRSs and their uncertainty measure methods, we have presented a method for decision-making to IMSIS. In this decision-making method for IMSIS, we have analyzed three issues. (1) How to find the similarity degrees between two objects from IMSISs in the Pythagorean fuzzy settings. (2) How to obtain the Pythagorean fuzzy decisionmaking objects from IMSISs. (3) The following problem: if $X_{1}, X_{2}, \ldots, X_{r} \subseteq U$ ( $U$ is the finite universe of discourse) then find the best $X_{r}$, where the elements of $X_{r}$ are randomly selected and not repeated any other elements in $X_{r}$. The studies of this paper are focusing on the basis of the theoretical aspect and the general framework of decision-making process to the IMSIS of the proposed model and method. Therefore, it is recommended that the further improvement of the proposed method to apply more complexity decisionmaking problems in Garg [5-7,9,10], Mandal and Ranadive [27] and the real-life data be used to test the approach established in this paper.

Acknowledgements The authors would like to thank the Guest Editor Harish Garg and reviewers for their thoughtful comments and valuable suggestions.

\section{Compliance with ethical standards}

Conflict of interest Prasenjit Mandal and A. S. Ranadive declare that there is no conflict of interest.

Ethical approval This article does not contain any study performed on humans or animals by the authors.

Informed consent Informed consent was obtained from all individual participants included in the study.

Open Access This article is distributed under the terms of the Creative Commons Attribution 4.0 International License (http://creativecomm ons.org/licenses/by/4.0/), which permits unrestricted use, distribution, and reproduction in any medium, provided you give appropriate credit to the original author(s) and the source, provide a link to the Creative Commons license, and indicate if changes were made.

\section{References}

1. Atanassov KT (1986) Intuitionistic fuzzy sets. Fuzzy Sets Syst 20(1):87-96

2. Azam N, Zhang Y, Yao JT (2017) Evaluation functions and decision conditions of three-way decisions with gametheoretic rough sets. Eur J Oper Res 261:704-714

3. Du WS, Hu BQ (2016) Dominance-based rough set approach to incomplete ordered information systems. Inf Sci 346:106-129

4. Duda RO, Hart PE (1973) Pattern classification and scene analysis. Wiley, New York

5. Garg H (2016) A novel accuracy function under interval-valued Pythagorean fuzzy environment for solving multicriteria decision making problem. J Intell Fuzzy Syst 31:529-540

6. Garg H (2017) Confidence levels based Pythagorean fuzzy aggregation operators and its application to decision-making process. Comput Math Organ Theory 23:546-571

7. Garg H (2017) Generalized Pythagorean fuzzy geometric aggregation operators using Einstein t-norm and t-conorm for multicriteria decisionmaking process. Int J Intell Syst 32:597-630

8. Garg H (2018) Generalised Pythagorean fuzzy geometric interactive aggregation operators using Einstein operations and their application to decision making. J Exp Theor Artif Intell. https:// doi.org/10.1080/0952813X.2018.1467497

9. Garg H (2018) Hesitant Pythagorean fuzzy sets and their aggregation operators in multiple attribute decision-making. Int J Uncertain Quantif 8:267-289

10. Garg H (2018) Linguistic Pythagorean fuzzy sets and its applications in multiattribute decisionmaking process. Int J Intell Syst. https://doi.org/10.1002/int.21979

11. Garg H (2018) New exponential operational laws and their aggregation operators for interval-valued Pythagorean fuzzy multicriteria decision-making. Int J Intell Syst. https://doi.org/10.1002/int. 21966

12. Garg H (2018) Some methods for strategic decisionmaking problems with immediate probabilities in Pythagorean fuzzy environment. Int J Intell Syst. https://doi.org/10.1002/int.21949

13. Huang B, Li H, Feng G, Zhuang Y (2017) Inclusion measure-based multigranulation intuitionistic fuzzy decision-theoretic rough sets and their application to ISSA. Knowl Based Syst. https://doi.org/ 10.1016/j.knosys.2017.10.003

14. Huang B, Wu W, Yan J, Li H, Zhou X (2018) Inclusion measurebased multi-granulation decision-theoretic rough sets in multiscale intuitionistic fuzzy information tables. Inf Sci. https://doi. org/10.1016/j.ins.2018.08.061

15. Li HX, Zhang LB, Zhou XZ, Huang B (2017) Cost-sensitive sequential three-way decision modeling using a deep neural network. Int J Approx Reason 85:68-78

16. Li JH, Huang CC, Qi JJ, Qian YH, Liu WQ (2017) Three-way cognitive concept learning via multi-granularity. Inf Sci 378:244263

17. Li WW, Huang ZQ, Jia XY, Cai XY (2016) Neighborhood based decision-theoretic rough set models. Int J Approx Reason 69:1-17

18. Liang D, Xu Z, Liu D, Wu Y (2018) Method for three-way decisions using ideal topsis solutions at Pythagorean fuzzy information. Inf Sci 435:282-295

19. Liang DC, Liu D (2015) Deriving three-way decisions from intuitionistic fuzzy decision-theoretic rough sets. Inf Sci 300:28-48

20. Liang DC, Xu ZS, Liu D (2017) Three-way decisions based on decision-theoretic rough sets with dual hesitant fuzzy information. Inf Sci 396:127-143

21. Liang JY, Li R, Qian YH (2012) Distance: a more comprehensible perspective for measures in rough set theory. Knowl Based Syst $27: 126-136$ 
22. Lin G, Liang J, Qian Y, Li J (2016) A fuzzy multigranulation decision-theoretic approach to multi-source fuzzy information systems. Knowl Based Syst 91:102-113

23. Liu D, Liang D, Wang C (2016) A novel three-way decision model based on incomplete information system. Knowl Based Syst 91:3245

24. Mandal P, Ranadive AS (2017) Multi-granulation bipolar-valued fuzzy probabilistic rough sets and their corresponding three-way decisions over two universes. Soft Comput. https://doi.org/10. 1007/s00500-017-2765-6

25. Mandal P, Ranadive AS (2018) Decisiontheoretic rough sets under Pythagorean fuzzy information. Int J Intell Syst 33(4):818-835

26. Mandal P, Ranadive AS (2018) Fuzzy multi-granulation decisiontheoretic rough sets based on fuzzy preference relation. Soft Comput. https://doi.org/10.1007/s00500-018-3411-7

27. Mandal P, Ranadive AS (2018) Hesitant bipolar-valued fuzzy sets and bipolar-valued hesitant fuzzy sets and their applications in multi-attribute group decision making. Granul Comput. https://doi. org/10.1007/s41066-018-0118-1

28. Mandal P, Ranadive AS (2018) Multi-granulation fuzzy decisiontheoretic rough sets and bipolar-valued fuzzy decision-theoretic rough sets and their applications. Granul Comput. https://doi.org/ 10.1007/s41066-018-0111-8

29. Mandal P, Ranadive AS (2018) Multi-granulation interval-valued fuzzy probabilistic rough sets and their corresponding three-way decisions based on interval-valued fuzzy preference relations. Granul Comput. https://doi.org/10.1007/s41066-018-0090-9

30. Pawlak Z (1982) Rough sets. Int J Inf Comput Sci 11:341-356

31. Peng X, Yuan H, Yang Y (2017) Pythagorean fuzzy information measures and their applications. Int J Intell Syst 32(10):991-1029

32. Sun B, Ma W, Zhao H (2014) Decision-theoretic rough fuzzy set model and application. Inf Sci 283:180-196
33. Wang ZH, Wang H, Feng QR, Shu L (2015) The approximation number function and the characterization of covering approximation space. Inf Sci 305:196-207

34. Yager RR (2014) Pythagorean membership grades in multicriteria decision making. IEEE Trans Fuzzy Syst 22(4):958-965

35. Yao YY, Wong SKM (1992) A decision theoretic framework for approximating concepts. Int J Man Mach Stud 37:793-809

36. Zhang C, Li D, Ren R (2016) Pythagorean fuzzy multi-granulation rough set over two universes and its applications in merger and acquisition. Int J Intell Syst 31(9):921-943

37. Zhang C, Li D, Ren R (2016) Pythagorean fuzzy multigranulation rough set over two universes and its applications in merger and acquisition. Int J Intell Syst 31(9):921-943

38. Zhang HY, Yang SY, Ma JM (2016) Ranking interval sets based on inclusion measures and applications to three-way decisions. Knowl Based Syst 91:62-70

39. Zhang X, Xu Z (2014) Extension of topsis to multiple criteria decision making with Pythagorean fuzzy sets. Int J Intell Syst 29:1061-1078

40. Zhang XX, Chen DG, Tsang ECC (2017) Generalized dominance rough set models for the dominance intuitionistic fuzzy information systems. Inf Sci 378:1-25

41. Zhao XR, Hu BQ (2015) Fuzzy and interval-valued decisiontheoretic rough set approaches based on the fuzzy probability measure. Inf Sci 298:534-554

42. Zhao XR, Hu BQ (2016) Fuzzy probabilistic rough sets and their corresponding three-way decisions. Knowl Based Syst 91:126-142

Publisher's Note Springer Nature remains neutral with regard to jurisdictional claims in published maps and institutional affiliations. 